\title{
Mémoires du livre
}

Studies in Book Culture

\section{L'extrait et ses fonctions dans la presse d'Ancien Régime}

\section{Timothée Léchot}

Volume 8, numéro 2, printemps 2017

Le livre et le journal : croisements, prolongements et transformations The Book and the Periodical: Intersections, Extensions and Transformations

URI : https://id.erudit.org/iderudit/1039696ar

DOI : https://doi.org/10.7202/1039696ar

Aller au sommaire du numéro

Éditeur(s)

Groupe de recherches et d'études sur le livre au Québec

ISSN

1920-602X (numérique)

Découvrir la revue

Citer cet article

Léchot, T. (2017). L'extrait et ses fonctions dans la presse d'Ancien Régime.

Mémoires du livre / Studies in Book Culture, 8(2).

https://doi.org/10.7202/1039696ar
Résumé de l'article

Aux XVII ${ }^{\mathrm{e}}$ et XVIII ${ }^{\mathrm{e}}$ siècles, les extraits constituent le type d'article privilégié de nombreux périodiques qui, sur le modèle du Journal des savants, rendent compte des livres parus dans les différents domaines des lettres. En étudiant la manière dont les journalistes francophones définissent l'extrait et ses principales fonctions dans l'espace médiatique, nous verrons que ce genre est au centre d'un faisceau de tensions qui mobilisent une éthique de la lecture et une déontologie journalistique en plein développement. On se demande alors notamment si l'extrait doit contenir une critique impartiale, ou s'il peut servir d'instrument de promotion au libraire, voire à l'auteur de l'ouvrage recensé. De telles réflexions situent l'extrait à l'intersection de l'histoire du livre, de la presse, de la lecture et de la critique.

Tous droits réservés @ Groupe de recherches et d'études sur le livre au Québec, 2017

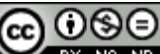




\section{MÉMOIRES DU LIVRE \\ STUDIES IN BOOK CULTURE}

\section{L'EXTRAIT ET SES FONCTIONS DANS LA PRESSE D'ANCIEN RÉGIME}

Timothée LÉCHOT

Université de Bâle

Aux XVII ${ }^{e}$ et XVIII ${ }^{e}$ siècles, les extraits constituent le type d'article privilégié de nombreux périodiques qui, sur le modèle du Journal des savants, rendent compte des livres parus dans les différents domaines des lettres. En étudiant la manière dont les journalistes francophones définissent l'extrait et ses principales fonctions dans l'espace médiatique, nous verrons que ce genre est au centre d'un faisceau de tensions qui mobilisent une éthique de la lecture et une déontologie journalistique en plein développement. On se demande alors notamment si l'extrait doit contenir une critique impartiale, ou s'il peut servir d'instrument de promotion au libraire, voire à l'auteur de l'ouvrage recensé. De telles réflexions situent l'extrait à l'intersection de l'histoire du livre, de la presse, de la lecture et de la critique.

In the $17^{\text {th }}$ and $18^{\text {th }}$ centuries, extracts were a type of article favoured by many periodicals that reviewed books in the different fields of Letters, following the example of the Journal des savants. By considering how Frenchspeaking journalists defined the extract and its main functions within media, we will see that this genre was at the centre of conflicting interests that involved a reading ethics and a developing ethics of journalism. Writers of the time wondered if the extract should contain an impartial review, or if it could serve as a promotional tool for the bookseller or even for the author of the book. Such reflections locate the extract at the junction of the history of books, of journalism, of reading, and of literary criticism. 
À l'intersection de l'histoire du livre, de la lecture, de la critique et de la presse périodique, se situe l'histoire d'une pratique, celle de l'«extrait». Dans son acception journalistique, le mot désigne aux XVII et XVIII ${ }^{e}$ siècles un compte rendu d'ouvrage, c'est-à-dire le résumé ou l'analyse d'un livre et quelquefois d'un périodique, d'un spectacle ou d'une autre forme de production culturelle. Variant selon la nature de la publication recensée, ces textes contiennent généralement les références bibliographiques de celle-ci, l'exposition de son projet, l'abrégé de ses différentes parties et des observations stylistiques, à quoi peuvent s'ajouter ou se substituer des citations parfois très étendues, ainsi que les réflexions et les éventuelles critiques du journaliste ${ }^{1}$. Consubstantiels aux journaux dits « littéraires» ou savants, qui sont souvent des mensuels ${ }^{2}$ et qui embrassent toute l'étendue des connaissances, les extraits forment une des principales catégories d'articles dès la naissance de cette classe de périodiques au XVII e siècle, à côté des dissertations et autres textes érudits de première main. Ils offrent de précieuses informations sur la réception des œuvres au moment de leur parution et c'est dans cette perspective que les historiens ont l'habitude de les consulter.

Cependant, si l'extrait constitue un ancêtre commun de notre critique journalistique et de nos recensions scientifiques, l'impression de familiarité qu'il entretient avec ces dernières peut conduire à des interprétations biaisées. En effet, les attentes qu'on projette sur ce type d'articles sous l'Ancien Régime varient d'un journal à l'autre, de même que les enjeux économiques, sociaux, moraux et épistémiques qui sous-tendent leur rédaction. L'extrait engage une éthique de la lecture et une déontologie journalistique qui embrassent cette pratique tout en la dépassant, et dont l'évolution n'est pas linéaire. En portant un regard large sur la presse d'Ancien Régime, il est possible de situer l'extrait dans le faisceau des tensions qui caractérisent ses principales fonctions au sein de l'espace médiatique.

Pour mener cette enquête dans la presse francophone, nous nous concentrerons moins sur les comptes rendus eux-mêmes que sur les partis pris explicites des journalistes à leur égard, souvent détaillés dans les prospectus, avertissements et autres textes liminaires qui accompagnent le lancement d'un nouveau périodique. Les notices du Dictionnaire des journaux dirigé par Jean Sgard $^{3}$ nous ont appris que, entre les déclarations d'intention 
du rédacteur ou du libraire et le contenu réel du périodique, il pouvait y avoir un fossé vertigineux. Parue récemment, une étude stimulante de Suzanne Dumouchel tient compte de cet écart. Elle se fonde sur cinq journaux du XVIII e siècle pour montrer le rôle du périodique littéraire dans l'émergence d'une culture et d'une communauté de lecteurs virtuelles ${ }^{4}$. Au centre de cette recherche, le compte rendu de livre est saisi dans le système de communication que forme chaque journal. Loin de se résumer à un simple outil d'information, il concourt à modifier subrepticement les habitudes de lecture et d'écriture, ce que Dumouchel révèle en examinant les principes avancés par les journalistes et leur application dans une large gamme de comptes rendus. La synthèse que nous proposons ici suit une méthode différente par la mise en série des textes préfaciels et par la réintroduction de la figure du libraire dans l'économie de l'extrait. Ainsi, ce n'est pas l'histoire des différents journaux cités que nous nous proposons d'éclairer, mais bien celle d'un genre et d'une pratique, en déployant l'éventail de ses représentations dans le discours journalistique. Nous nous arrêterons en particulier sur les défis qui accompagnent le résumé des œuvres, sur la relation de complémentarité ou de substitution que le compte rendu entretient avec le livre, sur la confrontation entre ses aspects promotionnels et l'impératif d'impartialité, et sur l'articulation entre une analyse neutre et un jugement critique.

\section{La moelle et le squelette}

La fonction la plus évidente de l'extrait est celle d'offrir un abrégé de l'ouvrage, soit en résumant systématiquement son contenu, soit en tirant de lui les éléments les plus dignes d'être divulgués et conservés. Cette fonction est aussi la plus ancienne. Avant d'investir les journaux du XVII ${ }^{e}$ siècle, l'extrait constitue une pratique savante personnelle. Comme le rappelle Élisabeth Décultot ${ }^{5}$, on annote et on résume des ouvrages depuis l'Antiquité. Érigé en art, ce geste connait d'importants développements à la Renaissance, tandis que les études humanistes se répandent et que la quantité de livres imprimés augmente exponentiellement. Si les recueils de lieux communs et les collections d'adversaria prolifèrent dans les bibliothèques manuscrites, certains font l'objet d'une publication dès le XVI siècle. Lorsque des journaux comme les Philosophical Transactions à Londres et le Journal des savants à Paris publient, dès les années 1660, des comptes rendus d'ouvrages, ils ne se substituent pas à la tradition humaniste de 
l'extrait manuscrit, mais ils l'adaptent à un médium dont l'enjeu principal est communicationnel : les résumés de livres sortent de la sphère privée pour s'adresser potentiellement à toute la communauté savante, sinon à d'autres strates du public ${ }^{6}$. Présidant à la rédaction des extraits personnels, le "principe de productivitê $»$, selon lequel l'art du résumé doit permettre à un auteur de rassembler des matériaux pour construire ses propres textes, cède le pas à des impératifs inédits qui modèlent l'extrait journalistique et lui définissent une existence indépendante de son parent manuscrit. Diffuser efficacement les connaissances, rendre accessibles les travaux spécialisés, distinguer les meilleurs ouvrages et consigner le progrès des lettres comptent parmi les raisons d'être de cette nouvelle forme d'extrait, telle que les journalistes la présentent.

Le débat reste ouvert sur la meilleure manière de procéder. Le Journal des savants, qui suit une périodicité plus ou moins hebdomadaire à ses débuts ${ }^{8}$, est le premier journal à proposer régulièrement des « $\operatorname{extraits}^{9}$ » de livres nouveaux dès sa création en 1665 . Il restera un point de repère essentiel pour les faiseurs d'extraits jusqu'à la fin du XVIII e siècle. Ses premiers comptes rendus sont plutôt des annonces; ils déclinent le titre, l'imprimeur et le format du livre, avant d'en donner un aperçu très sommaire, assorti de quelques remarques qui le situent au sein de la production littéraire, par exemple pour en montrer l'utilité ou l'originalité. Parmi les nombreux émules de ce périodique, le Nouveau Journal des savants lancé à Rotterdam en 1694 témoigne déjà d'une insatisfaction à l'égard des extraits trop secs. Dans l'avertissement de la première livraison bimestrielle, apparait la métaphore du squelette qui hantera longtemps le discours sur l'extrait :

[...] l'on estime qu'une simple analyse des écrits des Sçavans, toute instructive qu'elle est, ne sçauroit guéres plairre; non plus qu'un squelet n'est guéres agréable à voir, quoy qu'il nous fasse bientôt connoitre tous les ossemens d'un animal, en nous les présentant dans leur propre situation : Nôtre Auteur [...] se disposera [...] à revêtir ce squelet, \& à ajoûter à l'analyse, qu'il fera des livres nouveaux, quelques reflexions; sans se détourner pourtant, le moins du monde, de son principal dessein, qui est de donner une juste idée des ouvrages qu'il touchera ${ }^{10}$.

Ancrée profondément dans l'imaginaire journalistique, l'image du squelette est toujours convoquée une soixantaine d'années plus tard, dans le Journal 
britannique $^{11}$, et le stigmate de la sécheresse reste le principal défaut du genre dans l'article EXTRAIT, s. m. (Belles-Lettr.), de l'Encyclopédie, rédigé par Jean-François Marmontel ${ }^{12}$. Dans les deux cas, le caractère décharné des comptes rendus est attribué à une complaisance pour l'analyse impersonnelle et froide au détriment des remarques et des réflexions du journaliste. Développant une véritable poétique de l'extrait, Marmontel avance une seconde raison : la pusillanimité des journalistes qui ne prennent pas le risque de donner leur opinion. Pour un ouvrage philosophique ou historique, on peut éventuellement se contenter d'« exprimer la substance de l'ouvrage, en présenter les raisonnemens ou les faits capitaux dans leur ordre \& dans leur jour ${ }^{13} »$, mais Marmontel privilégie ce qu'il appelle l'extrait "discuté », c'est-à-dire un article qui offre quelque chose de plus qu'un résumé. Selon l'encyclopédiste, qui sera bientôt le nouveau directeur du Mercure de France ${ }^{14}$, «l'analyse la plus exacte $\&$ la mieux détaillée sera toûjours un rapport insuffisant ${ }^{15} \%$. La primauté des réflexions (personnelles) sur l'analyse (objective) continuera d'être revendiquée par des journalistes jusqu'à la fin de l'Ancien Régime. En 1783, par exemple, le critique suisse Henri-David Chaillet croit se distinguer du «simple Journaliste ${ }^{16}$ » en laissant libre cours aux digressions que lui inspireront les livres dans le Nouvean Journal de littérature et de politique de l'Europe, et, en effet, l'extrait constitue souvent chez lui le prétexte au déploiement de longues dissertations.

Ainsi, la fonction analytique qui semblait définir l'extrait satisfait rarement les rédacteurs et les commentateurs. L'extrait doit être à la fois document et œuvre, résumé fidèle et article partiellement original. C'est dans le même esprit qu'on accuse parfois les faiseurs d'extraits de se complaire dans un rôle de copiste - critique qu'on brandit aussi contre les pratiques manuscrites de l'ars excerpendi - sous prétexte de donner des analyses minutieuses ${ }^{17}$. Le reproche n'épargne pas un périodique de référence comme le Journal des savants ${ }^{18}$, mais il menace à plus forte raison la catégorie des journaux qui, au XVIII ${ }^{e}$ siècle, se spécialisent dans la compilation et le résumé d'articles tirés des autres périodiques, et qui publient donc des extraits d'extraits. Comptant parmi eux, l'Esprit des journaux, lancé en 1772 par un imprimeur liégeois, s'explique ainsi l'échec de son prédécesseur de Mannheim le Journal des journaux (1760). Dans ce périodique-ci, on ne présentait que "quelques tristes débris du squelette même d'un livre», alors que la 
lettre-préface du nouveau journal promet avec une ironie qui souligne le caractère usé du débat « des extraits pleins de fraicheur \& d'embonpoint $1^{9}$ ».

À la même époque, le journaliste français Jean-Pierre-Louis de Luchet définit à son tour les qualités d'une bonne analyse au seuil de ses Nouvelles de la République des lettres : à la précision, à la clarté et à la fidélité du journaliste s'ajoutent les devoirs de comparer l'ouvrage avec d'autres publications touchant à la même matière, de relever les erreurs, d'éclaircir les points douteux et de combattre ce qui semble nuire à la morale ou au goût, le tout appuyé par des raisonnements et des faits. Ce protocole nécessite du temps : Luchet dénonce les « extraits précipités ${ }^{20}$ » qui n'inspirent au lecteur aucune confiance et qui empêchent de faire sentir toutes les nuances de l'ouvrage. Il rappelle ici un écueil du journalisme littéraire et savant qui a émergé au XVII siècle. En 1686, à Amsterdam, la Bibliothèque universelle et historique avoue déjà que, écrasés par la quantité et la longueur des volumes à recenser, les journalistes ont pris la mauvaise habitude «de parcourir la Préface \& la Table, \& de faire quelques raisonnemens là-dessus ${ }^{21} »$. Vingt-cinq ans plus tard, cette fois à La Haye, le Journal littéraire revient sur ce problème pour demander que les journalistes lisent les livres d'un bout à l'autre, la plume à la main ${ }^{22}$. Loin de disparaitre au XVIII siècle $^{23}$, l'accusation de superficialité devient un lieu commun dont s'amuse en 1752 le journaliste huguenot Jean Rousset de Missy, installé dans les Provinces-Unies. Faisant parvenir un livre à son confrère Prosper Marchand qui, plus jeune, a justement travaillé pour le Journal littéraire, il accompagne son envoi d'un commentaire amicalement moqueur: "Je vous ai envoyé la Refutation allemande de Mr. Frantz. parce que je crois que vous souhaitiez la voir pour en articuler, le format, les chapitre [sic], les Pages \&c comme il arrive souvent aux Journalistes ne fut-ce que pour faire connoitre qu'ils ont effectivement vû le Livre² ${ }^{24}$.

Une éthique de lecture incombe donc au journaliste. En définitive, celui-ci remplit la fonction d'un «médiateur entre les auteurs \& le public ${ }^{25}$ », comme l'écrit Marmontel dans l'Encyclopédie. Le syndrome du squelette compromet cette besogne de médiation en affadissant l'extrait et en vidant pour ainsi dire le livre recensé de sa substantifique moelle. Aussi les journalistes insistent-ils fréquemment sur la nécessité de saisir la substance de l'ouvrage plutôt que de présenter mécaniquement chacune de ses parties. Voir "presque d'un seul coup d'œil le dessein, la conduite \& les plus beaux endroits d'un Auteur»: telle est par exemple l'intention d'un mensuel 
hollandais en 1687, l'Histoire des ouvrages des savants ${ }^{26}$. Les bons extraits contiennent «en racourci toute la substance des meilleurs Ouvrages de Science ${ }^{27}$ », clame-t-on également au seuil d'un périodique parisien spécialisé dans les mathématiques et la physique: le journal est ainsi comparé au cabinet de curiosités qui expose un abrégé du monde. Dans le Journal littéraire, on estime même que la partie dévoile le tout, c'est-à-dire que la recension, si elle touche à l'essentiel de l'œuvre, permet aux lecteurs savants de deviner le reste. Voir «tout un Livre d'un seul coup d'œil28 ", telle est la vertu magique de l'extrait.

Une fois constatée, l'instantanéité quasi photographique de l'extrait ennoblit le travail de dissection auquel se livrent les journalistes. Le genre de l'extrait s'écarte d'une simple pratique bibliographique pour soutenir un rapprochement avec les arts visuels. C'est du moins ce qu'exprime le mathématicien genevois Louis Bertrand, un contributeur occasionnel de la Nouvelle Bibliothèque germanique, dans le préambule d'un compte rendu de 1756 :

S'il faut qu'un Extrait soit au Livre original, ce qu'une Estampe est à un Tableau, un Ouvrage que la plupart des Lecteurs trouveront avoir besoin de commentaire, n'est pas fort susceptible de ce qu'on nomme Extrait. Car, les couleurs exceptées, l'Estampe contient tout ce qu'offre le Tableau, \& l'imagination peut aisément suppléer le coloris. Il faudroit donc comparativement que l'Extrait d'un Ouvrage en contînt toute la substance, \& développât les raisonnemens sur lesquels on s'y appuye, au point qu'une pénétration ordinaire pût aller d'une proposition à l'autre sans s'égarer, \& sans trop regretter les liaisons qu'on se seroit donné la liberté d'omettre ${ }^{29}$.

En substituant la métaphore de la gravure à celle du squelette, Bertrand cerne élégamment les forces et les limites du genre. L'extrait écarte le superflu, mais sa lisibilité demeure tributaire de facteurs exogènes: la prédisposition de l'œuvre originale au résumé et l'imagination reconstructive du lecteur. Incidemment, le Genevois soulève une autre question qu'on pourrait formuler ainsi : à tout prendre, l'estampe la mieux faite invite-t-elle à découvrir son modèle ou peut-elle tenir lieu du tableau, sinon lui devenir préférable? 


\section{Un guide d'achat ou un substitut}

Comme l'estampe, qui permet de diffuser largement et à moindres frais la copie d'une œuvre peinte réduite à son expression essentielle, l'extrait remplit la fonction évidente et généralement valorisée de faire connaître un livre à une large échelle. Non seulement un ouvrage est susceptible de trouver plusieurs recenseurs, mais un même extrait peut être republié dans plusieurs périodiques, avec ou sans l'aveu de son auteur, sans parler des autres formes de reprises (traduction, citation, résumé) ou des éventuelles réactions qu'il va susciter. La circulation des extraits et des autres articles savants favorise le partage des connaissances au sein de la République des lettres - les commentateurs manquent rarement de le souligner -, mais elle recèle également des enjeux économiques pour l'éditeur du journal, pour le libraire qui vend le livre et pour le public qui constitue la clientèle de l'un et de l'autre. Dès lors, il y a deux manières abondamment discutées d'envisager les fonctions de l'extrait: on regarde celui-ci comme un guide d'achat pour la bibliothèque personnelle du lecteur ou comme un substitut du livre.

Les deux termes de ce débat sont déjà exposés et neutralisés à la naissance de l'extrait. Dans une lettre-préface de «L'imprimeur au lecteur », le Journal des savants se flatte en effet d'avoir une double utilité :

Je crois qu'il y a peu de personnes qui ne voient que ce Journal sera utile à ceux qui acheptent des Livres; puis qu'ils n'en achepteront point qu'ils ne connoissent auparavant : \& qu'il ne sera pas inutile à ceux mesme qui n'ont pas moyen d'en achepter; puis que sans les achepter, ils ne laisseront pas d'en auvoir une connoissance generale ${ }^{30}$.

Plutôt qu'un substitut, l'extrait se présente comme un expédient pour la frange du public la moins fortunée. Cependant, la longueur des comptes rendus va augmenter avec le temps et la fonction de substitution va prendre le dessus, d'après certains observateurs. À La Haye, L'Europe savante évoque en 1718 l'admiration qu'on portait jadis au Journal des savants, un livre " qui seul pouvoit en quelque maniere tenir lieu de tous les autres ${ }^{31} »$. Cette idée n'est peut-être pas aussi exagérée qu'il n'y parait. Plus tard dans le siècle, Rousset de Missy évoque à son correspondant Marchand un ouvrage consacré au naturaliste Buffon. Il ne connait le livre en question «que par les extraits qui ont été dans le Journal des savans ${ }^{32}$ », mais cela lui suffit pour 
condamner toute la philosophie du naturaliste. Il déduit en effet des extraits que «le systeme de Buffon [est] le plus boufon que j'aye lû (passez le jeu de mot ${ }^{33} \gg$. Quoi qu'il en soit, on sent bien le paradoxe : si un journal savant peut satisfaire tous les besoins du lecteur, celui-ci n'a plus d'intérêt à enrichir sa bibliothèque, et le marché du livre, dont le journal dépend directement, s'en trouve dangereusement perturbé.

En conséquence, l'argument commercial de la substitution n'est jamais présenté sans nuance par les journalistes. D'après certains périodiques spécialisés qui concentrent leurs forces sur une aire géographique bien définie, l'extrait offre une réponse au problème très concret de l'acquisition d'ouvrages publiés à l'étranger. Imprimée à Genève entre 1728 et 1734 et consacrée aux productions savantes d'Italie, la Bibliothèque italique remarque que la librairie de ce pays recèle des "Trésors ${ }^{34}$ » cachés et presque inaccessibles en raison de leur rareté, de leur prix, des relations peu développées entre les librairies française et italienne, et enfin de la langue qui constitue un obstacle. Seul le journal permet de donner quelque connaissance des livres «qu'on ne verra peut-être jamais ${ }^{35}$ ». Reprenant 20 ans plus tard la mission de la Bibliothèque italique, le Journal des savants d'Italie fait un pas de plus en présentant l'ignorance des publications étrangères comme une «honte » pour les gens de lettres et les journaux comme «un moïen de [s'en] garentir ${ }^{36} »$.

D'autres motifs de substituer l'extrait au livre méritent l'attention. À la même époque que le Journal des savants d'Italie, le Journal britannique aborde aussi la question sous l'angle du lecteur érudit et de ses besoins en information. Cependant, le rédacteur hollandais Mathieu Maty n'invoque pas la honte du savant face au livre inaccessible, mais la nécessité pour lui de rester focalisé sur une matière précise : celui qui travaille au progrès des sciences et des lettres doit «s'épargner des lectures, qui le détourneroient de son principal objet ${ }^{37} »$. Le journal participe donc d'une hygiène de lecture et il ne remplace les livres qu'à l'extérieur du domaine de spécialisation du savant. En revanche, pour "celui qui se contente d'effleurer les sciences", c'est-à-dire l'homme du monde, l'extrait vaut tout simplement mieux que le livre: cette catégorie de lecteurs «souhaite qu'on pique sa curiosité; il veut qu'on l'amorce par des objets, qui changent souvent \& qu'il saisisse sans effort ${ }^{38} »$. On tient des propos similaires en 1760 dans la préface du Journal 
ecclésiastique à l'égard des hommes d'Église, principaux destinataires du périodique, qui ne sont pas nécessairement des érudits :

Toutes les Sciences y seront exposées dans un détail suffisant pour en être instruit \& être en état d'instruire les autres. Avec ce secours, les Ecclésiastiques pouront s'occuper dans leur solitude, raisonner sur ces Sciences \& en parler avec avantage dans les Conférences qu'ils tiennent entr'eux ${ }^{39}$.

Ces propos sur l'usage du journal par les non-savants laissent entrevoir la fonction sociale de l'extrait, signalée dès 1713 par le Journal littéraire : s'il n'est pas possible d'acquérir une connaissance profonde des sciences à travers les extraits, on peut du moins y puiser de quoi soutenir une conversation superficielle, comme on l'attend d'un honnête homme dans une tradition de la sociabilité mondaine qui remonte au siècle antérieur. À côté des mondains et des curieux, ce sont encore les jeunes gens ${ }^{40}$ et les femmes ${ }^{41}$ que ciblent certains faiseurs d'extraits. Mieux qu'un succédané du livre érudit, le compte rendu est alors envisageable comme sa traduction ou sa réécriture vulgarisée ${ }^{42}$.

Mais quels que soient les catégories de lecteurs susceptibles de remplacer le livre par l'extrait et les motifs économiques, sociaux ou pédagogiques qui peuvent l'y inviter, des voix s'élèvent contre l'idée que l'extrait puisse se substituer au livre. La même année où le Journal ecclésiastique s'identifie à un « cabinet littéraire ${ }^{43}$ » autosuffisant, le Journal des journaux répond fermement par la négative aux questions suivantes :

Les ouvrages des Auteurs sont-ils détruits par les analises périodiques? Le Savant se contente-t-il de lire des extraits? Le Bibliotécaire acquiert-il moins? Le Lecteur est-il moins avide de belles éditions, \& des productions nouvelles? Le Libraire enfin embrasse-t-il moins dans son commerce toutes les parties de la terre où la lumière des Sciences a pénétré 44 ?

Dès le moment où l'on admet que le livre et l'extrait ne constituent pas un doublon, mais qu'ils entretiennent une relation de complémentarité, la fonction de guidage est invoquée presque systématiquement au moment de lancer un nouveau journal. 
Marchant dans les pas du Journal des savants au début du XVIII ${ }^{\mathrm{e}}$ siècle, L'Europe savante répète le double impératif d'informer les amateurs des sciences et des arts des progrès qu'on y fait et de leur éviter des dépenses inutiles. "C'est un préservatif contre le fard des Titres ${ }^{45}$ »: l'extrait, contrairement au relevé bibliographique ou au catalogue de libraire, dévoile le contenu qui se cache véritablement derrière un titre accrocheur. Souvent repris, l'argument du titre trompeur apparaît notamment dans l'Histoire littéraire de l'Europe (1726-1727) attribuée à Justus Van Effen, un célèbre journaliste hollandais. Mais Van Effen précise aussi que l'extrait, s'il suit promptement la parution du livre, guide l'acheteur sans attendre le verdict de la «voix publique ${ }^{46}$ » qui peut mettre du temps à se prononcer. $A$ contrario, pour Jacob Vernes, c'est l'opinion publique que le journaliste s'efforce de corriger. En effet, dans le Choix littéraire de ce rédacteur genevois, on lit en 1755 qu'un homme prudent «ne composeroit sa Bibliothéque que d'un petit nombre d'ouvrages choisis, qui ne devroient leur réputation, ni au charme de la nouveauté, ni au caprice de la mode, ni à la cabale des protecteurs et des amis ${ }^{47}$ ». Il met en garde contre la «vogue $e^{48}$ » épidémique qui accompagne parfois une nouvelle parution et dont l'extrait raisonné constitue l'antidote.

Qu'on ouvre encore la Critique désintéressée ${ }^{49}$, la Gazette littéraire et universelle de l'Europe $e^{50}$ ou le Journal de politique et de littérature ${ }^{51}$, tout le monde est d'accord : l'extrait permet avant tout de fuir les mauvais livres. Il ne fait aucun doute que cette fonction réponde aux attentes du lectorat. En 1752, par exemple, Rousset de Missy explique dans sa correspondance privée qu'il s'est épargné un achat inutile grâce à la lecture d'un extrait ${ }^{52}$. Inversement, au milieu de la décennie suivante, un lecteur anonyme du Journal belvétique prend la parole pour dire qu'il s'est fié à un article du périodique au moment d'acquérir un volume de poésies, mais qu'il a été trompé sur la qualité supposée de celles$\mathrm{ci}^{53}$. Comme ce lecteur en a fait l'expérience, l'extrait n'est pas un guide d'achat infaillible. Personne n'ignore d'ailleurs que des facteurs externes peuvent infléchir l'opinion du rédacteur. Soit qu'ils dénigrent leurs concurrents, soit qu'ils cherchent à prouver leur intégrité, les journalistes rappellent souvent les tentations auxquelles leur activité de critique les soumet : favoriser l'ouvrage d'un ami, déprécier celui d'un ennemi, relayer la publicité d'un libraire, accepter de l'argent ou des cadeaux... Intimement liée à la fonction de guidage, la question de l'impartialité et de ses limites doit à son tour arrêter notre attention. 


\section{La promotion et l'autopromotion au risque de la partialité}

Si le journalisme peut être théorisé par Marmontel comme un acte de médiation, il constitue également un instrument de médiatisation. Les rédacteurs et les auteurs de l'Ancien Régime font l'expérience de ses vertus promotionnelles pendant les premières décennies où la presse savante se développe. À la fois auteur et journaliste, le marquis Jean-Baptiste Boyer d'Argens reconnaît l'importance des périodiques pour assurer le succès de ses œuvres littéraires : ayant déjà trop d'ennemis parmi les journalistes, il résiste à la tentation de dénigrer la Bibliothèque française dans ses Lettres Juives, en vertu d'une critique positive dont cette fiction épistolaire a bénéficié54. Les intérêts respectifs des auteurs, des libraires-éditeurs et des journalistes orientent donc parfois le discours critique et minent souterrainement l'impartialité des articles. Cependant, à une époque où les fonctions attachées à ces différents métiers ne sont pas toujours clairement définies ni séparées, les aspects promotionnels de l'extrait ne contredisent pas obligatoirement la recherche d'une certaine objectivité chez les acteurs des périodiques.

En particulier, le journal littéraire peut servir de vitrine au catalogue du libraire qui l'édite. On l'a déjà noté, par exemple, à l'égard du Nouveau Journal helvétique (1769-1779) imprimé par la Société typographique de Neuchâtel, laquelle profite du périodique pour présenter ses propres publications ou les ouvrages dont elle relaie la diffusion ${ }^{55}$. Mais le phénomène est bien antérieur. À la fin du XVII ${ }^{\mathrm{e}}$ siècle, il n'y a rien de choquant à ce que l'auteur de l'Histoire des ouvrages des savants, le Français exilé à Rotterdam Henri Basnage de Beauval, dépende étroitement de son libraire Reinier Leers. D’un côté, Basnage profite de "l'abondance des meilleurs Livres ${ }^{56}$ » qui se trouvent chez Leers pour choisir les titres à recenser. De l'autre, le libraire s'immisce dans la préface du journaliste pour annoncer qu'il tâchera d'obtenir promptement un certain nombre des livres évoqués par Basnage dans le journal, s’ils ne sont pas déjà sur ses rayons, de manière à ce que les lecteurs puissent se les procurer. Le journal s'expose donc sans inconvénient comme une entreprise commerciale qui fonctionne en vase clos, ce qui n'empêche pas Basnage d'ambitionner une parfaite équité dans sa critique. 
En raison du coût des livres et des conditions difficiles de leur diffusion à une large échelle, il est évident que l'éditeur d'un journal a besoin d'entretenir de bons contacts non seulement avec les hommes de lettres, mais encore avec le monde de la librairie. Ce besoin est parfois formulé dans les avis des journaux. En 1726, par exemple, l'Histoire littéraire de l'Europe de Van Effen lance un appel ouvert aux libraires pour les prier d'envoyer franc de port «les Livres nouveaux, dont ils croiront l'Extrait nécessaire ${ }^{57}$ ». On prend aussi la mesure du phénomène dans les correspondances privées. Entre les années 1730 et les années 1750, celle de Prosper Marchand contient plusieurs lettres qui accompagnent ou promettent l'envoi d'un livre, à condition que le destinataire rédige un extrait journalistique du volume concerné58.

Pourtant, dès la première moitié du XVIII ${ }^{e}$ siècle, la fonction promotionnelle des journaux est pointée du doigt par des hommes du métier. Lorsqu'il s'adresse au marquis d'Argens, Marchand décrie en 1736 la relation de servitude que les auteurs de la Bibliothèque raisonnée entretiennent avec leur libraire : «Ce n'est pas un seul Savant qui compose ce Journal : ils sont sept ou huit, tous très humbles Esclaves de leur Libraire, ne louant que ce qui lui plait et déchirant avec Fureur et Mauvaise-Foi tout ce qui ne lui convient point ${ }^{59}$.» Une critique semblable avait déjà été émise quelques années auparavant, cette fois publiquement, dans les Lettres sérieuses et badines, un périodique catholique dont l'épître dédicatoire satirique est adressée en 1729 à la très protestante Bibliothèque raisonnée. Point par point, on y attaque la ligne éditoriale de ce périodique qui ne gratifierait d'une critique positive que les livres «imprimez ou débitez chez les Wetsteins \& Smith ${ }^{60} »$, ses deux libraires amstellodamois. Pourtant, les Lettres sérieuses et badines ne rechigneront pas à recenser à leur tour les publications haguenoises de leur propre libraire Johannes Van Duren ${ }^{61}$. Les concessions des journalistes aux professionnels de la librairie sont plus faciles à dénoncer qu’à éviter.

Distinct de la publicité éditoriale, d'ailleurs aisée à déceler par le lecteur attentif, un autre phénomène promotionnel mériterait une étude spécifique, dans la mesure où l'on sous-estime certainement son étendue: l'autorecension. Comme le laissent entrevoir les propos de Marchand sur la Bibliothèque raisonnée, un journal savant n'est pas forcément l'œuvre d'un seul journaliste. Et même lorsque le nom d'un périodique est attaché à celui d'un rédacteur particulier, celui-ci reproduit souvent les articles que des 
collaborateurs ou de simples lecteurs lui envoient. Les contributeurs sont difficiles à identifier derrière l'anonymat quasi généralisé. Or, à l'égard des extraits, ces voix anonymes peuvent compter les auteurs mêmes des livres analysés. Faire le compte rendu de son propre ouvrage n'est pas rare : non seulement des cas peuvent être identifiés ponctuellement, mais on trouve dans plusieurs périodiques des appels ouverts à l'autorecension. D'un côté, cette pratique offre à l'auteur un certain contrôle sur la réception de ses œuvres. De l'autre, elle compromet encore la légitimité du journal en tant que guide d'achat et instance critique.

L'autorecension a ses avantages : elle garantit au lecteur un résumé exact et elle le protège des journalistes qui se contenteraient de parcourir le livre. En introduisant cette idée dans sa première livraison de 1686, la Bibliothèque universelle et bistorique tente d'anticiper les bénéfices qu'elle en tirera et les risques qui en découleront. D'abord, sa préface avance que les journalistes doivent se mettre dans la peau des auteurs pour analyser leurs ouvrages sans partis pris idéologiques et pour viser un même degré de fidélité. Ensuite, ce sont les auteurs eux-mêmes qui sont invités à envoyer leurs comptes rendus :

$[\ldots]$ lors que quelcun craindra qu'on ne prenne pas assez bien sa pensée, ou qu'on n'insiste pas assez sur les endroits les plus considerables de son ouvrage, il n'a qu'à prendre la peine d'en faire l'extrait lui-méme, \& de nous l'envoier. On l'inserera tout entier dans cette Bibliotheque, à condition néanmoins qu'on ne répondra point de la fidelité de l'extrait, si ce n'est qu'on ait vû l'ouvrage méme, \& qu'on les ait pû comparer ensemble ${ }^{62}$.

Et lorsque l'ouvrage n'aura pu être consulté, on n'autorisera pas l'auteur à être trop affirmatif quant aux vérités qu'il croira avoir démontrées : les deux journalistes Jean Leclerc et Jean Cornand de La Crose retoucheront les extraits en conséquence, s'ils l'estiment nécessaire.

Même précaution dans le périodique français connu sous les noms de Mémoires ou Journal de Trévoux, lancé par des pères jésuites en 1701. Cette publication qui veut rassembler "des extraits de tous les Livres de Science » imprimés en Europe estime que "personne, ordinairement parlant, n'est capable de faire mieux l'extrait d'un livre que celui qui l'a composé63». Elle enjoint donc aux auteurs de préparer leurs textes et elle promet «de les y 
inserer tels qu'ils les enverront: après cependant qu'on les aura comparez avec les Ouvrages mêmes, pour s'assûrer que ces extraits sont fidelles ${ }^{64}$ ». Ce rôle d'éditeur, les journalistes l'abandonneront déjà en 1712 en refusant désormais avec fermeté les extraits rédigés par les auteurs ${ }^{65}$. Cinquante ans plus tard, le Journal britannique convient toujours que les auteurs sont les mieux placés pour abréger leurs ouvrages, mais il réaffirme simultanément l'importance du journaliste: la distance qu'il entretient avec le livre lui permet de clarifier les passages obscurs et de relever des faiblesses que l'auteur est susceptible de (se) cacher $^{66}$.

Contemporaine du Journal britannique, la Bibliothèque impartiale de Jean Henri Samuel Formey clame hautement, comme son nom l'indique, sa prétention d'impartialité en matière de critique : aucun motif étranger aux ouvrages ne doit interférer avec les jugements du journaliste dans ses extraits. Malgré cette déclaration de principe, les auteurs et les libraires sont priés d'envoyer leurs publications. Les auteurs peuvent même joindre les extraits de leurs livres, qui ne seront pas signés, mais présentés comme des articles reçus et éventuellement accompagnés d'un commentaire ${ }^{67}$. Formey utilise le même procédé dans la Nouvelle Bibliothèque germanique, à laquelle il travaille de 1746 à 1759, et où apparaît de manière récurrente la mention « cet extrait nous a été fourni» en note de bas de page. Le naturaliste suisse Élie Bertrand, par exemple, saisit l'opportunité de faire lui-même la promotion de ses traités dans ce dernier journal ${ }^{68}$. Quant à Johann Albrecht Euler, il reçoit la commande de rédiger la critique d'un livre de son propre père, le célèbre mathématicien bâlois Leonhard, et il s'exécute avec application ${ }^{69}$.

La tension entre la revendication d'impartialité et une ouverture à l'autopromotion se perçoit encore dans un journal plus tardif, la Gazette littéraire et universelle de l'Europe publiée à Lausanne entre 1768 et 1769. En dépit de son désir affirmé d'« équité», d'«impartialité» et de «désintéressement ${ }^{70}$ », le libraire-journaliste François Grasset supplie les savants de recenser les ouvrages auxquels ils ont donné naissance, sans marquer la moindre inquiétude par rapport aux dérives publicitaires d'une telle pratique. Par contraste, d'autres journaux prennent leurs distances avec toute forme de promotion des livres ou des auteurs. La Critique désintéressée de François Bruys fait de la distinction entre auteurs et journalistes son fer de lance en 1730. Elle définit l'utilité d'une critique «sans égard, ni pour les Auteurs, ni pour les Libraires ${ }^{71} »$ et elle dénonce la partialité du Journal des 
savants et de la Bibliothèque raisonnée $e^{72}$. À la même époque, on lit aussi dans l'Histoire littéraire de l'Europe l'importance de choisir les ouvrages à présenter une des missions les plus délicates du journaliste - sans se laisser influencer ni par «l'amour propre des Auteurs» ni par «l'avare liberalité des Libraires $^{73}$ ». Idem en 1738: la Nouvelle Bibliothèque on histoire littéraire stigmatise le journaliste qui devient «le Fauteur de l'Avidité de certains Libraires $^{74} \gg$.

Indépendamment de la personnalité qui se cache derrière un extrait, la critique injuste fait du tort à la République des lettres, ce qu'admet non seulement l'Histoire littéraire de l'Europe, mais aussi la Bibliothèque germanique qui craint la «désunion ${ }^{75}$ » parmi les gens de lettres. Plus accusateurs, les Mémoires secrets du marquis d'Argens considèrent en 1737 la partialité des journalistes comme le «vice interne ${ }^{76}$ » des milieux savants. Aussi le crédo d'impartialité est-il certainement le principal lieu commun du discours métajournalistique sous l'Ancien Régime. Il se décline en une infinité de variantes. On prône un juste milieu entre la censure sévère et la flatterie servile dans l'Histoire des ouvrages des savants ${ }^{77}$; on fait profession d'exactitude et de bonne foi dans le Journal littéraire ${ }^{78}$; on invoque une parfaite neutralité à l'égard des partis (religieux, politiques, intellectuels) qui règnent en Angleterre dans la Bibliothèque anglaise ${ }^{79}$; on s'engage à une critique juste et ménagée dans un périodique plus généraliste comme le Mercure de France ${ }^{80}$; on rêve toujours, dans la seconde moitié du XVIII siècle, que le journaliste ne laisse rien deviner de sa religion, de sa patrie et de son caractère ${ }^{81}$. Résumant un siècle de débat en 1779, le Journal de littérature, des sciences et des arts enserre entre quatre mots la définition d'une bonne critique journalistique : «impartiale, motivée, instructive, honnête ${ }^{82}$ ».

Comme le rappelle le spécialiste de l'histoire de la presse Alexis Lévrier, l'objectivité des journalistes «n'est souvent qu'une posture ${ }^{83}$ », les rédacteurs français et hollandais se livrant volontiers à la polémique. Il arrive qu'on crédite un journal d'une impartialité louable, comme par exemple la Bibliothèque italique, félicitée de maintenir une parfaite neutralité en matière de disputes religieuses malgré le protestantisme affiché de ses auteurs ${ }^{84}$. À la fin des années 1720, cette même Bibliothèque italique reconnaît cependant que tous les journalistes de l'Europe ont pris le même engagement d'impartialité, mais que "presque tous l'ont violé en quelque occasion ${ }^{85}$ ». Cette observation s'est muée en fatalité lorsque, une vingtaine d'années plus tard, 
le Journal britannique avoue honnêtement: «Ceux qui ont prescrit à un Journaliste d'être parfaitement impartial connoissent trop l'humanité, pour espérer qu'il puisse jamais s'en trouver de pareil ${ }^{86}$.

En dernière analyse, l'impartialité est une clause centrale du contrat qu'établit le journaliste avec son lecteur, mais elle constitue un élément perturbateur des relations que ce même journaliste entretient avec les libraires et les auteurs dont son activité dépend directement. Si le faiseur d'extraits critique ses fournisseurs de livres, fût-ce légitimement, il s'isole et s'attire à son tour la vindicte. Mais s'il ménage complaisamment ses amis ou ses correspondants, il perd l'estime du lecteur qui ne manque pas d'exprimer sa déception et que personne n'oblige à renouveler son abonnement. Difficile de réaliser le vœu d'un Denis Diderot qui, dans l'Encyclopédie, demande au journaliste que «son intérêt soit entierement séparé de celui du libraire \& de l'écrivain ${ }^{87} »$. Les conditions ne sont pas réunies pour que tous les acteurs du livre et du journal subsument leurs intérêts respectifs sous un système de critique autonome sur le plan intellectuel et moins étroitement connecté au circuit économique. Pourtant, dans leur effort de résoudre le paradoxe de l'impartialité, les journalistes de l'Ancien Régime testent des solutions qui concourent à définir cet espace critique. Nous allons le suggérer en évoquant trois problématiques qui intéressent l'histoire de l'extrait : la réflexion sur la nécessité de juger un livre, l'invention de comités de lecture et l'affirmation de l'auctorialité du journaliste.

\section{Le jugement : « vanité ridicule » ou « obligation sacrée »?}

La solution la plus simple au problème de l'impartialité consiste en un refus de juger les ouvrages. Une approche entièrement descriptive du compte rendu ne garantit pas une absolue neutralité - le journaliste reste maitre de choisir les livres dont il parle -, mais elle écarte d'un même geste le blâme et la louange. Éviter ces deux écueils, telle est déjà la «ferme résolution ${ }^{88}$ » de Leclerc et Cornand de La Crose dans la Bibliothèque universelle et historique: l'enjeu consiste à présenter les idées des auteurs sans exprimer d'avis sur elles. Plus explicitement, des Essais de littérature qui paraissent en 1702 associent les louanges des journalistes à leur vénalité, estimant que les rédacteurs tirent un profit matériel des critiques positives dont ils gratifient un auteur. Le réquisitoire est violent : «Je ne sai quels gens érigez, on ne sait 
comment, en Arbitres des Ouvrages d'esprit font, à leur fantaisie, le Panégyrique ou la Satyre des Auteurs, \& ne nous disent presque rien des Livres, dont il est vrai aussi qu'ils sont tres-incapables de juger ${ }^{89}$. » Persuadé que les jugements des journalistes ont causé la disparition de nombreux livres, l'abbé français Anthelme Tricaud présente son périodique comme un contrepoison aux effets néfastes de la critique : il n'exhumera que des livres anciens, rares ou singuliers, et dans tous les cas presque inconnus. En 1730, un article du Nouvelliste du Parnasse vante l'absence de critique directe qui caractérise le Journal des savants, quoique, la même année, la Critique désintéressée que nous avons citée plus haut tienne des propos très différents sur ce même journal. Pour les responsables du Nouvelliste Pierre-François Guyot Desfontaines et de François Granet, le Journal des savants a le mérite de laisser aux lecteurs attentifs le soin de décider si le livre est bon ou mauvais d'après l'analyse dépassionnée qu'il en donne ${ }^{90}$. Ainsi, lorsqu'il fait confiance au discernement du public, le journaliste peut abandonner sa verve de critique sans perdre totalement sa fonction de guide.

Quoi qu'il en soit, se donner «l'autorité [...] sublime » de juger un ouvrage apparaît comme une "vanité ridicule" dès le XVII e siècle. En 1684, on trouve ces expressions dans les Nouvelles de la République des lettres de Pierre Bayle, dont les activités journalistiques marqueront durablement le monde de la presse, et dans l'Histoire des ouvrages des savants trois ans plus $\operatorname{tard}^{91}$. Plus qu'un garde-fou contre la tentation de la flatterie, le refus d'une critique qui tranche en faveur ou aux dépens d'un auteur participe d'un ethos journalistique qui implique une certaine humilité. De ce point de vue, condamner un livre est un acte presque sacrilège. Beaucoup plus tard, en 1774, un autre journaliste célèbre le martèlera sans ambages. Bien qu'on se souvienne de lui comme d'un champion de la polémique littéraire, Simon Linguet regrette en effet l'époque de Bayle et s'étonne du «despotisme qu'exercent si hardiment, \& avec tant d'impunité dans une République libre,

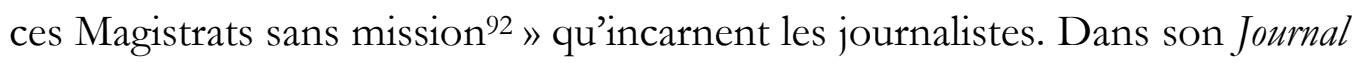
de politique et de littérature, Linguet ne considère pas ceux-ci comme des juges. Ils peuvent tenter de pressentir les décisions du public à l'égard des nouvelles publications, mais ils ne doivent hasarder une opinion qu'avec une extrême prudence.

Pourtant, les avantages de la critique sont difficiles à ignorer, si bien que de nombreux commentateurs plaident en faveur de son développement. Laisser 
libre cours à son jugement saupoudre d'un précieux sel les extraits, ce qui permet de corriger leur fadeur ${ }^{93}$. Encadrée par la morale, la critique contribue au développement de la société et à l'adoucissement des mœurs, deux vertus qui légitiment cette activité chez les journalistes ${ }^{94}$. Mais surtout, on comprend l'utilité de perfectionner, à travers l'institution journalistique, un instrument de distinction spécialisé. S’il est écrit par des personnalités fiables, le journal est capable d'offrir aux auteurs une reconnaissance publique qui crée une émulation et qui stimule le progrès des lettres. Pour L'Europe savante, prendre le parti de juger est une hardiesse qu'on doit s'autoriser dans un journal. Non seulement « tout Lecteur a ce droit ${ }^{95}$ », mais la critique profitera à tout le monde, y compris aux auteurs dont les livres auront déplu. La fonction critique prime même sur la fonction informative de résumé puisque, comme l'annonce le fondateur du journal Thémiseul de Saint-Hyacinthe, les livres sans intérêt ou trop volumineux pour se prêter à un extrait seront l'objet d'une «simple indication, ou un jugement justifié par quelques passages ${ }^{96} \gg$. Nous sommes en 1718. En 1726, un autre pas est franchi dans l'Histoire littéraire de l'Europe, qui présente les louanges comme «le prix du merite \& du travail » et comme des insignes « sacrées ${ }^{97}$ » que le journaliste ne distribue qu'avec la plus grande délicatesse :

$[\ldots]$ les accorder à ceux qui en sont indignes, ou confondre dans les mêmes éloges les excellens Livres \& les bons Ouvrages, c'est prostituer les lauriers, les profaner, leur ôter ce qu'ils ont de flatteur, en dégouter les Savans, \& embarasser les Lecteurs, qui ne savent plus ce qu'ils doivent penser des Livres, parce que l'Auteur du Journal n'y met pas la moindre difference ${ }^{98}$.

N'en déplaise plus tard à Linguet, les journalistes ne sont pas des «Magistrats sans mission », mais bien plutôt les interprètes des muses et les mandataires d'un office quasi sacré.

L'argumentaire en faveur de la critique va encore se développer au cours du XVIII ${ }^{e}$ siècle où se succèdent des figures marquantes de journalistes, telles que l'abbé Desfontaines et Élie Fréron. Rédigé par l'abbé Jean-Baptiste Grosier, un ancien collaborateur de Fréron, le Journal de littérature, des sciences et des arts est introduit en 1779 par des «Idées préliminaires sur la Critique » qui permettent de mesurer le chemin parcouru. La critique n'est rien de moins qu'une «nécessité ${ }^{99}$ ». Si certains journalistes craignaient jadis qu'une critique trop blessante conduise à la faillite de la République des lettres, 
Grosier pense au contraire que l'intransigeance des jugements est salvatrice : «De quelle corruption prochaine ne seroient point menacées les Lettres, si une Critique sévère ne fixoit l'opinion publique sur le mérite ou les défauts de cette foule d'écrits; $[\ldots]^{100} »$. Le journalisme littéraire apparait alors dans toute sa gloire : loin de relever de «la basse littérature », il forme un genre exigeant - «l'un des plus difficiles ${ }^{101}$ » - et il suppose des qualités nombreuses (réflexion mûre, justesse, tact, esprit d'analyse, goût, large érudition). Ces considérations débouchent sur l'exposé d'un pacte journalistique entre le critique et le lecteur, qui manifeste une vibrante modernité en même temps que l'inertie de certaines problématiques. Le passage mérite d'être cité in extenso:

La censure littéraire est, en quelque sorte, un ministère public, fondé sur la confiance. Depuis que le goût des connoissances est devenu celui de presque tous les âges $\&$ de tous les rangs, le ton général des sociétés semble exiger au moins une teinture des lettres; il est indispensable de paroitre instruit. Mais pour se mettre en état de raisonner avec quelque justesse de toutes les productions nouvelles, il faudroit avoir le loisir \& le courage de dévorer, chaque année, une multitude immense de volumes, \& je conviens que cette tâche seule est capable d'effrayer le lecteur le plus intrépide. L'amour propre \& la paresse ont donc fait recourir aux Journaux. On se promet qu'un écrivain périodique exposera, dans une analyse exacte, ce que contiennent les ouvrages, \& qu'en les soumettant à l'œil sévère de la critique, il saura les apprécier à leur juste valeur. Cette déférence du public pour le goût \& les lumières d'un Journaliste, lui annonce l'importance de ses fonctions. Ses jugemens, adoptés par une multitude de lecteurs influeront sur l'opinion générale: il est devenu, en quelque sorte, l'arbitre du succès des ouvrages $\&$ de la réputation de leurs auteurs. Malheur à l'Ecrivain qui, dans une pareille position, ne sentiroit pas l'obligation sacrée d'être juste, \& qui, d'une main vénale, distribueroit le blâme ou l'éloge! Il manque à un devoir que la probité même lui impose, \& son infidélité dégénère en un véritable abus de confiance ${ }^{102}$.

Comme ministère fondé sur la confiance et comme tribunal d'arbitrage du succès littéraire, la critique acquiert dans ce plaidoyer le statut d'une véritable institution d'intérêt public. Elle se voit dotée d'une autorité suffisante pour 
ne rendre de comptes ni aux libraires ni aux auteurs, mais seulement à son principal destinataire, le lectorat.

Bien avant d'en arriver là, une autre stratégie est testée pour garantir l'impartialité de l'extrait. L'idée est introduite à l'aube du XVIII siècle dans le Journal des savants par son directeur du moment, l'abbé Jean-Paul Bignon, qui forme un comité de lecture en s'inspirant du fonctionnement des académies. Se réunissant une fois par semaine, cette «Compagnie de Gens de Lettres » trouve son sens dans le fait «qu'une seule personne ne peut pas suffire à la lecture de tous les Livres, \& à faire les Extraits dont le Journal doit estre composé103 ». Une structure plus élaborée est mise en place dans le Journal littéraire de 1713. Derrière celui-ci œuvre non seulement Van Effen, mais «une espéce de Société » formée de «plusieurs personnes de differens Païs ${ }^{104} »$. Les membres de cette société se répartissent la préparation des extraits en fonction de leurs domaines respectifs de compétence : "Chacun d'eux travaillera sur les matiéres \& les Sciences qu'il a le plus étudiées, \& ils ont réglé la maniére de faire examiner les Extraits \& autres Pieces qui doivent entrer dans ce Journal, par un certain nombre choisi d'entr'eux pour cela ${ }^{105}$. » La relecture par des pairs évite ainsi des jugements trop personnels; elle contribue également à l'uniformité et à la qualité des textes. Observée par Ioana Galleron Marasescu, cette «méthode nouvelle d'évaluation des œuvres ${ }^{106} \gg$ n'empêche pas le Journal littéraire d'en appeler à la collaboration des libraires et des auteurs pour faire connaitre leurs productions. Le procédé est repris peu de temps après par L'Europe savante. Le travail collectif d'une «Société composée de Gens, dont chacun s'applique à quelque Science particuliere ${ }^{107}$ », vaut mieux qu'un journaliste unique, parce que cette société protège la fidélité des articles. Pour ce faire, l'extrait est soumis à «un examen critique, dont les contradictions amenent à l'impartialité108 ». La description du procédé reste elliptique. On lit seulement les injonctions suivantes : « [...] qu'un autre examine l'Extrait sur le Livre; \& que le Livre \& l'Extrait soient encore examinez par toute une Societé109. » L'évaluation du livre et de l'extrait par un groupe doit rendre le jugement plus solide, et non conduire à un plat consensus. Elle ne compromet pas la mission critique de L'Europe savante, mais elle la couvre au contraire d'un vernis d'objectivité.

Ce qu'on appelle aujourd'hui le peer review trouve donc son origine dans l'Ancien Régime et touche d'abord les recensions de livres. Néanmoins, ce 
système d'évaluation ne dispose pas encore de règles explicites et partagées; il ne garantit pas l'anonymat des pairs, ni leur neutralité, ni même leur statut d'experts. Reste que la crédibilité des journaux en profite. La Critique désintéressée souligne positivement le fait que, dans le Journal littéraire, aucun extrait ne paraisse être le travail d'un seul auteur ${ }^{110}$. Quant à la compagnie du Journal des savants, elle est encore louée vers 1730 dans le Nouvelliste du Parnasse:

La forme [du Journal des savants] n'en a pas toûjours été la même. Dans les commencemens on se contentoit d'indiquer l'usage des livres modernes, \& d'en porter des jugemens sans aucuns extraits; mais peu à peu le Journal est devenu analytique. [...] les extraits sont lûs avant que d'être imprimez, dans une assemblée à laquelle préside un de ces hommes rares [une note précise: M. l'Abbé Bignon] que le Ciel fait naitre de tems en tems, pour encourager les Lettres ${ }^{111}$.

Malgré ces essais concluants, les comités de lecture d'extraits semblent former une mode qui n'est pas destinée à perdurer. En 1756, Pierre Rousseau, fondateur du Journal encyclopédique, demande encore que ses collaborateurs consultent « les Hommes les plus celebres ${ }^{112}$ » - ou du moins des personnalités externes au journal - pour profiter de leurs lumières sur les ouvrages parus, sinon pour soumettre directement à leur approbation les extraits à publier. Mais de telles déclarations se raréfient dans les préfaces des journaux ultérieurs.

Parallèlement à l'affermissement de cette critique qu'on pourrait qualifier d'objective, en tant qu'elle cherche à établir des jugements fiables et fondés, l'idée d'une critique subjective et assumée comme telle s'affirme progressivement. Suzanne Dumouchel remarque que «la forme périodique favorise l'expression personnelle des rédacteurs ${ }^{113} »$, qui instaurent un dialogue en différé avec le lecteur. L'échange intersubjectif apparait comme un élément structurel du périodique littéraire, en particulier lorsque celui-ci adopte la fiction d'une correspondance entre une figure de journaliste qui s'exprime à la première personne et un destinataire anonyme auquel le lecteur peut s'identifier. De son côté, Alexis Lévrier observe aussi le «triomphe d'un journalisme d'expression individuelle ${ }^{114}$ » pendant la première moitié du XVIII ${ }^{e}$ siècle. En particulier, on développe alors «un journalisme de critique littéraire délibérément subjectif ${ }^{115}$ », malgré l'idéal 
d'impersonnalité et de rigueur qui, hérité de la presse savante du XVII siècle, exclut théoriquement toute polémique des journaux.

De fait, le caractère subjectif des jugements critiques compte d'abord parmi les reproches qui entretiennent un scepticisme à l'égard des extraits. Le Journal littéraire remarque en 1713 qu'il y a autant de tours d'esprit parmi les journalistes qu'il y a d'expressions différentes sur les visages. Un même livre peut impressionner les hommes de différentes manières, selon leur caractère, et chaque expérience de lecture est singulière. En admettant cela, la fonction de guide ou d'instrument de distinction dont s'habille l'extrait est compromise. Selon la société de rédacteurs qui s'occupe du Journal littéraire, promettre une bonne foi ne suffit pas : pour se faire une idée juste d'un livre, il est indispensable de comparer les différents extraits qui portent sur lui dans les différents périodiques. Chemin faisant, «chaque Lecteur pourra trouver facilement la face sous laquelle il aime a regarder un Ouvrage ${ }^{116} »$. La multiplication des journaux littéraires apparait dans cette perspective comme une évolution bénéfique de la presse.

L'argument fait mouche auprès de l'Histoire littéraire de l'Europe qui le répète ${ }^{117}$, mais il peut se retourner à son tour contre les périodiques. Tandis que le nombre de journaux littéraires suit une croissance exponentielle ${ }^{118}$, il semble en effet fastidieux (et onéreux) de procéder à la lecture de tous les extraits à chaque fois qu'on veut se faire une opinion. L'éphémère Fenille nécessaire, qui paraît au cours de l'année 1759 à Paris, remarque que les contradictions qui règnent entre les journaux à l'égard d'un même objet « ne laissent souvent au Lecteur que l'embarras de juger les jugemens mêmes ${ }^{119}$ ». À cet embarras, le Journal des journaux se propose de remédier en 1760. Ses articles étant basés sur ceux des autres journaux, il compte fondre les extraits en un seul pour réunir les suffrages et faire la synthèse de leurs éventuelles divergences : «Chacun pense \& juge comme il est affecté. Mais le Lecteur qui attend des Journalistes une décision qui le détermine, demeure indécis lorsqu'il aperçoit une si grande contrariété. Notre Journal vient à propos pour le décider ${ }^{120}$. » La même année, c'est aussi l'objectif d'un autre journal peu durable, Le Littérateur impartial ${ }^{121}$.

Avec plus de succès, dès la première moitié du XVIII ${ }^{e}$ siècle, des journalistes s'érigent en écrivains et s'imposent par leur style, leur liberté d'expression et leur forte personnalité littéraire, phénomène qu'on connait depuis les 
travaux pionniers d'Eugène Hatin ${ }^{122}$. On passe alors, pour ainsi dire, d'un journalisme de librairie à un journalisme d'auteur. La pratique traditionnelle de l'extrait se heurte au nouveau modèle, comme on le constate dans un différend entre le journaliste Rousset de Missy et le libraire Marc-Michel Rey, lequel assure à partir de 1749 la réimpression hollandaise du Journal des savants, augmentée d'articles originaux. Rousset écrit sa colère à Marchand :

Rey est un petit fat. [...] Je lui ai offert de lui envoyer de tems en tems quelques Morceaux sur des livres nouveaux pour ses additions au J. des Sav. il a eu l'impertinence de me répondre qu'il Les recevroit avec plaisir, mais que je ne devoit [sic] pas me formaliser, s'il ne se servoit pas de tous, ou s'il changeoit quelque chose dans les autres. Qu'il aille se faire dégraisser à Montfaucon! il n'aura pas une ligne de $\mathrm{moi}^{123}$.

Ce sont bien les prérogatives d'un auteur que défend ici le directeur de L'Épilogueur moderne et l'ancien collaborateur de la Bibliothèque raisonnée. Vers 1750, le journalisme littéraire participe de l'habitus des hommes de lettres. Dans la Bibliothèque impartiale, on lit que « les plus illustres Ecrivains » ont pris le soin «d'augmenter leur réputation par cet endroit ${ }^{124}$ ». Parmi beaucoup d'autres, on pourrait alléguer l'exemple de Diderot dont l'activité journalistique est connue ${ }^{125}$. Une anecdote est révélatrice de son engagement en la matière. En 1759, Diderot n'hésite pas à ajourner une rencontre avec sa maitresse Sophie Volland, parce qu'il consacre deux nuits consécutives à préparer des extraits de pièces de théâtre pour son ami Friedrich Melchior Grimm, principal rédacteur d'un célèbre périodique manuscrit, la Correspondance littéraire, philosophique et critique ${ }^{126}$.

Cependant, et les articles de Diderot suffiraient à le rappeler, la recherche d'une expression critique libérée bute contre les limites assignées à l'extrait traditionnel. Centré sur un seul livre dont on attend un exposé fidèle, l'extrait n'est pas un essai : il faut posséder l'art de la digression à la manière d'un Chaillet pour s'autoriser, d'une part, une lecture subjective du livre et pour développer, d'autre part, une réflexion singulière sur des questions d'esthétique, de poétique, de morale, de société, etc. ${ }^{127}$ Aussi le journalisme d'expression personnelle se présente-t-il comme une alternative bienvenue à la tradition des «bibliothèques » et autres journaux savants. En France, cette approche du journalisme correspond déjà à celle des périodiques mondains du XVII siècle : le Mercure galant de Jean Donneau de Visé, écrit à la première 
personne, entretient une impression de familiarité et d'intimité entre le journaliste et ses lecteurs. Au XVIII e siècle, le phénomène s'étend à d'autres sortes de périodiques. Importés de l'Angleterre et introduits en France par Marivaux, les «spectateurs» d'où émane la voix d'un énonciateur individualisé participent du nouveau paradigme ${ }^{128}$, de même que les journaux rédigés par lettres dont Fréron comptera parmi les promoteurs les plus notoires ${ }^{129}$. Les petites feuilles spectatoriales et le genre épistolaire autorisent en effet une grande variété de styles, un ton provocateur, une expression enjouée et une présentation déliée des livres. Le journaliste y gagne en liberté et le lecteur en plaisir. En 1731, au seuil d'une décennie qui verra les périodiques par lettres se multiplier, le jeune Nouvelliste du Parnasse distingue ainsi la lettre et l'extrait dans sa douzième livraison :

Notre but, comme vous sçavez, n’a jamais été de faire des extraits des livres nouveaux; nos lettres sont destinées à des réflexions sur les ouvrages d'esprit, \& sur d'autres, lorsqu'ils amenent l'occasion de dire des choses agréables ou curieuses. Ce n'est pas sans raison que nous avons choisi le genre épistolaire, outre que le stile en est libre \& aisé, certains tours qui lui sont familiers, donnent de l'éclat \& de la vivacité aux réflexions ${ }^{130}$.

Le chemin qui conduit à un journalisme plus subjectif semble donc s'écarter de la pratique de l'extrait. Pourtant, la lettre ne se distingue pas aussi radicalement du compte rendu que le Nowvelliste le suggère. Elle en absorbe les fonctions, et il n'est pas rare de trouver, à l'intérieur d'une lettre journalistique, un extrait en bonne et due forme qui résume, analyse, cite, commente le livre et décline toutes ses informations bibliographiques. On le constate par exemple dans l'Année littéraire (1754-1776), le journal le plus célèbre de Fréron qui, à travers le genre épistolaire, compte sur "la liberté de dire naturellement ce qu'il pense sur les nouveautés ${ }^{131} »$ sans perdre de vue le caractère informatif de l'extrait ${ }^{132}$.

\section{Conclusion}

Ainsi, l'extrait est un genre protéiforme et perméable à différents discours. S'il éclot dans la presse savante, il s'étend à d'autres approches du journalisme tournées vers un public moins érudit et attentives au divertissement du lecteur. Les postures des journalistes se construisent et s'éprouvent dans les définitions qu'ils en donnent et dans les fonctions qu'ils 
lui attribuent, ce qui fait de lui un indicateur précieux pour comparer les périodiques et pour cerner à la fois l'évolution et la diversification de ce médium. En tant qu'écrit squelettique, l'extrait représente un véritable défi rédactionnel pour les journalistes, qui doivent repenser l'alliance horatienne de l'utile et de l'agréable dans le cadre d'un texte tissé de commentaires et de citations, piste qui mériterait d'être suivie en isolant les nombreuses réflexions stylistiques des préfaces et des articles. En tant qu'œuvre de seconde main, basée sur une publication préexistante, le compte rendu place le journaliste au milieu d'un triangle dont les sommets sont l'auteur, le libraire et le lecteur. Dépendant de cette relation tripartite, le faiseur d'extraits a besoin de préciser la position qu'il y occupe et la manière dont il peut l'enrichir ou la rééquilibrer. Enfin, en tant que support de la critique littéraire en phase de constitution, l'extrait contribue à ériger le journalisme en profession distincte au sein des milieux littéraires et savants, avec une déontologie propre et une fonction publique reconnue.

Le champ d'investigation qu'ouvre l'extrait serait sans doute moins riche si ce type d'articles n'était pas soumis aux tensions, voire aux paradoxes dont nous avons tenté d'offrir un aperçu. Naissant pour la plupart au XVII siècle ou dans la première moitié du XVIII ${ }^{e}$, les débats relatifs aux comptes rendus ne débouchent pas sur une définition consensuelle du genre à la fin de l'Ancien Régime. Il serait possible de suivre leur évolution pendant la période révolutionnaire, à un moment où la presse se politise, et au cours du xix ${ }^{\mathrm{e}}$ siècle, qui verra la notion même d'« extrait » disparaître progressivement du discours journalistique francophone au profit d'autres termes comme « critique », « compte rendu » et, au xx ${ }^{\mathrm{e}}$ siècle, « recension ».

Timothée Léchot consacre ses recherches à la littérature française du XVIII siècle et à la presse périodique d'Ancien Régime. Dans ce domaine, il a notamment coédité en 2016 les actes d'un colloque consacré au Journal helvétique. Il a soutenu à l'Université de Neuchâtel une thèse de doctorat sur la poésie suisse d'expression française au XVIII' siècle et au début du XIXe, publiée en 2017 à la Librairie Droz (Genève). À l'Université de Bâle, il collabore actuellement à un projet de recherche et d'édition portant sur l'écrivain Jacques Delille et le phénomène de la poésie scientifique. 


\section{Notes}

1 Certains chercheurs distinguent les «extraits» (brefs), les «comptes rendus» (développés) et les «articles». C'est par exemple le cas de Daniel Roche dans Les circulations dans l'Europe moderne. XVII'-XVIII siècle [2003], s. l., Arthème Fayard, Pluriel, 2010, pp. 106-110. Or, cette distinction projette une typologie anachronique sur la presse d'Ancien Régime. Aux XVII et XVIII ${ }^{\mathrm{e}}$ siècles, on parle souvent d'« extrait » pour désigner ce que nous appelons aujourd'hui un «compte rendu» de livre, indépendamment de la longueur du texte concerné. En outre, le terme d'« article» ne renvoie pas à une catégorie définie de textes journalistiques; on s'en sert dans certains périodiques pour indiquer les différentes sections du numéro : article « Littérature », article " France », etc.

2 Pour Marmontel, en 1756, la présence d'extraits caractérise «les journaux \& autres ouvrages périodiques qui paroissent tous les mois, \& où l'on rend compte des livres nouveaux » (Jean-François Marmontel, EXTRAIT, s. m. (Belles-Lettr.), dans Denis Diderot, Jean le Rond D'Alembert (dir.), Encyclopédie ou dictionnaire raisonné des sciences, des arts et des métiers, par une société de gens de lettres, t. 6, Paris, Briasson, David, Le Breton, Durand, 1756, p. 334a).

${ }^{3}$ Jean Sgard (dir.), Dictionnaire des journaux (1600-1789), Paris, Universitas, 1991. Édition en ligne: "Dictionnaire des journaux 1600-1789. Édition électronique revue, corrigée et augmentée ", Lyon, Institut des sciences de l'homme, LIRE UMR 5611: http://dictionnaire-journaux.gazettes18e.fr (31 octobre 2016).

${ }^{4}$ Suzanne Dumouchel, Le Journal littéraire en France au dix-buitième siècle. Émergence d'une culture virtuelle, Oxford, The Voltaire Foundation, 2016.

5 «Introduction. L'art de l'extrait : définition, évolution, enjeux », dans Élisabeth Décultot (dir.), Lire, copier, écrire. Les bibliothèques manuscrites et leurs usages au XVIII siècle, Paris, CNRS Éditions, 2003, pp. 7-28.

6 Sur le journal d'Ancien Régime comme support de la communication savante, voir Jeanne Peiffer, Jean-Pierre Vittu, «Les journaux savants, formes de la communication et agents de la construction des savoirs (17e-18e siècles) ", Dix-huitième siècle, $\mathrm{n}^{\circ}$ 40, 2008, pp. 281-300.

7 Élisabeth Décultot, «Introduction. L'art de l'extrait : définition, évolution, enjeux », dans Élisabeth Décultot (dir.), Lire, copier, écrire. Les bibliothèques manuscrites et leurs usages an XVIII siècle, Paris, CNRS Éditions, 2003, p. 20. Ici et dans la suite de notre étude, le soulignement est dû à l'auteur du texte cité.

8 Voir Jean-Pierre Vittu, «Journal des savants (1665-1792, 1797)», dans Jean Sgard (dir.), Dictionnaire des journaux (1600-1789), Paris, Universitas, 1991, no 710.

9 Dans les premiers volumes du Journal des savants, le terme d'«extrait» s'applique prioritairement aux extraits de lettres et aux extraits d'autres journaux. Cependant, il s'étend progressivement aux comptes rendus de livres dans les années 1670 et 1680.

Vol. 8, n 2 | Printemps 2017

«Le livre et le journal : croisements, prolongements et transformations » 
10 "Le libraire au lecteur », Nouveau Journal des sçavans, dressé à Rotterdam, par le Sieur C*****, Rotterdam, Pierre Vander Slaart, janvier-février 1694, s. p.

11 Voir «Préface », Journal britannique, par M. Maty, Docteur en Philosophie \& en Médecine, La Haye, H. Scheurleer Junior, janvier 1750, p. III.

12 Jean-François Marmontel, EXTRAIT, s. m. (Belles-Lettr.), dans Denis Diderot, Jean le Rond D'Alembert (dir.), Encyclopédie ou dictionnaire raisonné des sciences, des arts et des métiers, par une societé de gens de lettres, t. 6, Paris, Briasson, David, Le Breton, Durand, 1756, pp. 334a-335b. Voir aussi l'entrée «Extrait» des Éléments de littérature (1787) de Marmontel (Sophie Le Ménahèze (éd.), Paris, Éditions Desjonquères, 2005, pp. 540-545).

13 Jean-François Marmontel, EXTRAIT, s. m. (Belles-Lettr.), dans Denis Diderot, Jean le Rond D'Alembert (dir.), Encyclopédie ou dictionnaire raisonné des sciences, des arts et des métiers, par une société de gens de lettres, t. 6, Paris, Briasson, David, Le Breton, Durand, 1756, p. 334a.

14 Voir Jacques Wagner, Marmontel journaliste et le Mercure de France (1725-1761), Grenoble, Presses universitaires de Grenoble, 1975.

15 Jean-François Marmontel, EXTRAIT, s. m. (Belles-Lettr.), dans Denis Diderot, Jean le Rond D'Alembert (dir.), Encyclopédie ou dictionnaire raisonné des sciences, des arts et des métiers, par une société de gens de lettres, t. 6, Paris, Briasson, David, Le Breton, Durand, 1756, p. 334b.

16 [Henri-David Chaillet], Prospectus d'un Nouveau Journal de littérature et de politique de l'Europe, et sur-tout de la Suisse, s. l. n. d., p. 3.

17 Voir Le Nouvelliste du Parnasse, ou réflexions sur les ouvrages nouveaux, Paris, Chaubert, t. 1, $12^{\mathrm{e}}$ lettre, 1731, pp. 277-300.

18 Voir «Article I. Journal des Savans, \&c. », Critique desinteressée des journaux littéraires et des ouvrages des savans. Par une Societé de Gens de Lettres, La Haye, Chrétien Van Lom, janviermars 1730, pp. 9-17.

19 "Lettre à $\mathrm{M}^{* * *} »$, L’Esprit des journaux, ouvrage périodique et littéraire, dédié à Son $A$. C. Monseigneur François-Charles des Comtes de Velbruck, Evêque \& Prince de Liege, \&c. \&. \&.c, Liège, J. J. Tutot, t. 1, juillet 1772, p. V.

20 [Préface sans titre], Nouvelles de la République des lettres, Lausanne, Société typographique, 1 ère partie, juillet 1775, p. 10.

21 "Préface », Bibliothèque universelle et historique de l'année M. D. C. LXXXVI., Amsterdam, Wolfganc, Waesberge, Boom, van Someren, t. 1, 168[6], s. p.

22 «Préface », Journal litéraire, La Haye, T. Johnson, t. 1, mai-juin 1713, p. XIII.

23 Voir par exemple les «Idées préliminaires sur la Critique » développées en 1779 dans la première livraison du Journal de littérature, des sciences et des arts; Par M. l'Abbé Grosier (Paris, Bureau du Journal), aux pages 3 à 33 .

24 Jean Rousset de Missy à Prosper Marchand, s. l., [29 septembre 1752], dans Christiane Berkens-Stevelinck, Jeroom Vercruysse (éd.), Le métier de journaliste an dix-buitième siècle. 
Correspondance entre Prosper Marchand, Jean Rousset de Missy et Lambert Ignace Douxfils, Oxford, The Voltaire Foundation, 1993, p. 155. L'ouvrage concerné est le suivant : Adam Wilhelm Franzen, Widerlegung der französischen Schrift : L'Homme machine, nebst dem Beweis der Gegensätze, Leipzig, Carl Ludwig Jacobi, 1749.

25 Jean-François Marmontel, EXTRAIT, s. m. (Belles-Lettr.), dans Denis Diderot, Jean le Rond D'Alembert (dir.), Encyclopédie ou dictionnaire raisonné des sciences, des arts et des métiers, par une société de gens de lettres, t. 6, Paris, Briasson, David, Le Breton, Durand, 1756, p. 335a.

26 "Préface », Histoire des ouvrages des sçavans, Par Monsr. B*** Docteur en Droit, Rotterdam, Reinier Leers, septembre 1687, s. $p$.

27 "Préface », Recherches de mathématique et de physique. Qui contient des Extraits des Journaux de France, \& des Païs étrangers, avec des Remarques sur ces Extraits. Par Monsieur Parent de l'Academie Royale des Sciences, Paris, Florentin Delaulne, Jean Jombert, t. 1, 1ère partie, 1705, p. 4.

28 «Préface », Journal litéraire, La Haye, T. Johnson, t. 1, mai-juin 1713, p. VII.

29 Louis Bertrand, "Article VII. Institutiones Calculi Differentialis [...]», Nowvelle Bibliothèque germanique, ou histoire littéraire de l'Allemagne, de la Suisse, es des Pays du Nord, par Mr. Samuel Formey, Amsterdam, Jean Schreuder, Pierre Mortier le Jeune, t. 18, 1 ère partie, janvier-mars 1756, pp. 101-102. L'ouvrage concerné est le suivant: Leonhard Euler, Institutiones calculi differentialis cum eius usu in analysi finitorum ac doctrina serierum. Auctore Leonhardo Eulero Acad. reg. scient. et eleg. litt. Boruss. directore. Prof. honor. Acad. imp. scient. Petrop. et Academiarum regiarum Parisinae et Londinensis Socio., Saint-Pétersbourg, Academiae imperialis scientiarum, 1755.

30 "L'imprimeur au lecteur", Le Journal des sçavans. Par le Sieur de Hedouville, Paris, Jean Cusson, 5 janvier 1665, s. $p$.

31 «Préface », L’Europe savante, La Haye, A. de Rogissart, t. 1, 1ère partie, janvier 1718, p. IV.

32 Jean Rousset de Missy à Prosper Marchand, s. l., [avant juin 1753], dans Christiane Berkens-Stevelinck, Jeroom Vercruysse (éd.), Le métier de journaliste au dix-buitième siècle. Correspondance entre Prosper Marchand, Jean Rousset de Missy et Lambert Ignace Douxfils, Oxford, The Voltaire Foundation, 1993, p. 223.

33 Jean Rousset de Missy à Prosper Marchand, s. l., [avant juin 1753], dans Christiane Berkens-Stevelinck, Jeroom Vercruysse (éd.), Le métier de journaliste au dix-huitième siècle. Correspondance entre Prosper Marchand, Jean Rousset de Missy et Lambert Ignace Douxfils, Oxford, The Voltaire Foundation, 1993, p. 223.

34 "Préface ", Bibliothèque italique on bistoire littéraire de l'Italie, Genève, Marc-Michel Bousquet \& Compagnie, t. 1, janvier-avril 1728, p. XIV.

35 "Préface", Bibliothèque italique on histoire littéraire de l'Italie, Genève, Marc-Michel Bousquet \& Compagnie, t. 1, janvier-avril 1728, p. XIV.

36 "Préface », Journal des savans d'Italie, Amsterdam, Aux dépens de la Compagnie, t. 1, 1748, p. IX.

Vol. 8, n 2 | Printemps 2017

«Le livre et le journal : croisements, prolongements et transformations » 
37 «Préface », Journal britannique, par M. Maty, Docteur en Philosophie \& en Médecine, La Haye, H. Scheurleer Junior, janvier 1750, p. V.

38 "Préface », Journal britannique, par M. Maty, Docteur en Philosophie \& en Médecine, La Haye, H. Scheurleer Junior, janvier 1750, p. V.

39 "Préface », Journal ecclésiastique, ou bibliothèque raisonnée des sciences ecclésiastiques. Ouvrage dédié à Son Altesse Monseigneur le Prince Louis de Rohan, Coadjuteur de l'Evêché de Strasbourg. Par M. l'Abbé Dinouart, Chanoine de l'Eglise Collégiale de Saint Benoît, \& de l'Académie des Arcades de Rome, t. 1, $1^{\text {ère }}$ partie, octobre 1760, p. III.

${ }^{40}$ Voir par exemple l'«Avertissement» de la Bibliothèque choisie, pour servir de suite à la Bibliothèque universelle. Par Jean le Clerc, Amsterdam, Henri Schelte, t. 1, 1703 [2nde édition], s. $p$.

41 Voir par exemple l'article «Des Feuilles Periodiques» dans les Éphémérides du citoyen, ou chronique de l'esprit national, Paris, Nicolas Augustin Delalain, t. 1, no 1, 4 novembre 1765, pp. 1-16.

42 Au-delà de la vulgarisation d'un contenu, le compte rendu participe d'une "pédagogie critique » que les périodiques littéraires prennent en charge et que Suzanne Dumouchel analyse aux pages 133 à 163 de Le Journal littéraire en France au dix-buitième siècle. Émergence d'une culture virtuelle, Oxford, The Voltaire Foundation, 2016. Sur les fonctions sociales du journal littéraire en tant que lieu d'échange culturel, voir les pages 269-273.

43 "Préface », Journal ecclésiastique, ou bibliothèque raisonnée des sciences ecclésiastiques. Ouvrage dédié à Son Altesse Monseigneur le Prince Louis de Rohan, Coadjuteur de l'Evêché de Strasbourg. Par M. l'Abbé Dinouart, Chanoine de l'Eglise Collégiale de Saint Benoît, \& de l'Académie des Arcades de Rome, t. 1, 1ère partie, octobre 1760, p. VIII.

44 « Discours préliminaire », Journal des journaux on précis des principaux Ouvrages Périodiques de l'Europe, par une Société de gens de Lettres, dédié à son Altesse sérénissime électorale palatine, Mannheim, Imprimerie électorale, t. 1, 1760, p. XIII.

45 «Préface », L’Europe savante, La Haye, A. de Rogissart, t. 1, 1 ère partie, janvier 1718, p. 17.

46 "Préface ", Histoire littéraire de l'Europe : contenant l'Extrait des meilleurs Livres; un Catalogue choisi des Ouvrages nouveaux; les Nouvelles les plus interessantes de la République des Lettres, \& les Pieces fugitives les plus curieuses, La Haye, M. G. de Merville, t. 1, janvier 1726, s. p.

${ }^{47}$ « Préface », Choix littéraire, Genève, Claude Philibert, t. 1, 1755, p. IV.

48 « Préface », Choix littéraire, Genève, Claude Philibert, t. 1, 1755, p. IV.

49 Voir "Préface ", Critique desinteressée des journaux littéraires et des ouvrages des savans, Par une Societé de Gens de Lettres, La Haye, Chrétien Van Lom, janvier-mars 1730, s. p.

${ }^{50}$ Voir "Préface des éditeurs », Gazette littéraire et universelle de l'Europe, qui contient l'Annonce \& les Extraits des principaux Livres qu'on y met an jour; avec divers morceaux sur l'Agriculture,

Vol. 8, nº 2 | Printemps 2017

« Le livre et le journal : croisements, prolongements et transformations » 
l'Oeconomie rurale, le Commerce, la Poesie, la Peinture, la Musique \&o la Sculpture, \&o. Eoc., Lausanne, François Grasset \& Comp., t. 1, 1768, pp. III-XIII.

${ }^{51}$ Voir [préface sans titre], Journal de politique et de littérature, contenant les principaux Evènemens de toutes les Cours; les Nouvelles de la République des Lettres, \&o., Bruxelles, s. n., t. 1, n 1, 25 octobre 1774, pp. 3-8.

52 Jean Rousset de Missy à Prosper Marchand, s. l., 13 décembre 1752, dans Christiane Berkens-Stevelinck, Jeroom Vercruysse (éd.), Le métier de journaliste an dix-buitième siècle. Correspondance entre Prosper Marchand, Jean Rousset de Missy et Lambert Ignace Douxfils, Oxford, The Voltaire Foundation, 1993, pp. 176-179.

53 Voir «Observations sur la nouvelle Traduction des Odes Sacrées, ou Psaumes de David, publiée par M. Garcin ", Journal helvétique ou recueil de pièces fugitives de litérature choisie; de Poësie, de Traits d'Histoire ancienne \& moderne; de Découvertes des Sciences \& des Arts; de Nouvelles de la République des Lettres; \& de diverses autres Particularités intèressantes \& curieuses, tant de Suisse, que des Pays Etrangers. Dedié au Roi, Neuchâtel, Imprimerie des éditeurs, avril 1765, pp. 369-380.

54 Voir la lettre de Jean-Baptiste Boyer, marquis d'Argens, à Prosper Marchand, s. l., [fin décembre 1736], dans Steve Larkin (éd.), Correspondance entre Prosper Marchand et le marquis d'Argens, Oxford, The Voltaire Foundation, 1984, pp. 91-93.

55 Voir l'« Introduction à l'histoire du Journal helvétique » dans Séverine Huguenin, Timothée Léchot (dir.), Lectures du Journal helvétique 1732-1782. Actes du colloque de Neuchâtel 6-8 mars 2014, Genève, Éditions Slatkine, 2016, pp. 23-82.

56 "Préface ", Histoire des ouvrages des sçavans, Par Monsr. B*** Docteur en Droit, Rotterdam, Reinier Leers, septembre 1687, s. $p$.

57 «Préface », Histoire littéraire de l'Europe : contenant l'Extrait des meilleurs Livres; un Catalogue choisi des Ouvrages nowveaux; les Nouvelles les plus interessantes de la République des Lettres, \& les Pieces fugitives les plus curieuses, La Haye, M. G. de Merville, t. 1, janvier 1726, s. p.

58 Voir les lettres suivantes : Jean-Baptiste Boyer, marquis d'Argens, à Prosper Marchand, s. l., [novembre 1738], dans Steve Larkin (éd.), Correspondance entre Prosper Marchand et le marquis d'Argens, Oxford, The Voltaire Foundation, 1984, pp. 185-188; Jean Rousset de Missy à Prosper Marchand, s. l., janvier 1749, p. 60, et Jean Rousset de Missy à Prosper Marchand, Anvers, 2 janvier [1751], pp. 111-114, dans Christiane Berkens-Stevelinck, Jeroom Vercruysse (éd.), Le métier de journaliste au dix-huitième siècle. Correspondance entre Prosper Marchand, Jean Rousset de Missy et Lambert Ignace Douxfils, Oxford, The Voltaire Foundation, 1993.

59 Prosper Marchand à Jean-Baptiste Boyer, marquis d'Argens, s. l., [janvier 1736], dans Steve Larkin (éd.), Correspondance entre Prosper Marchand et le marquis d'Argens, Oxford, The Voltaire Foundation, 1984, p. 53.

60 «A Messieurs les auteurs de la Bibliotheque raisonnée », Lettres sérienses et badines sur un Livre intitulé Etat présent de la République des Provinces-Unies. Par Mr. F. M. Janiçon. Et sur d'autres ouvrages, La Haye, Jean Van Duren, 1'̀re partie, 1729, p. VIII.

Vol. 8, nº 2 | Printemps 2017

«Le livre et le journal : croisements, prolongements et transformations » 
61 Voir Jean Sgard, «Lettres sérieuses et badines (1729-1740)», dans Jean Sgard (dir.), Dictionnaire des journaux (1600-1789), Paris, Universitas, 1991, no 834.

${ }^{62}$ "Préface », Bibliothèque universelle et historique de l'année M. D. C. LXXXVI., Amsterdam, Wolfganc, Waesberge, Boom, van Someren, t. 1, 168[6], s. $p$.

63 "Préface ", Mémoires pour l'histoire des Sciences et des beaux Arts. Recueillis par l'Ordre de Son Altesse Serenissime Monseigneur Prince Souverain de Dombes, Trévoux, Imprimerie de S. A. S., janvier-février 1701, s. $p$.

${ }^{64}$ "Préface », Mémoires pour l'histoire des Sciences et des beaux Arts. Recueillis par l'Ordre de Son Altesse Serenissime Monseigneur Prince Souverain de Dombes, Trévoux, Imprimerie de S. A. S., janvier-février 1701, s. p.

65 Voir Pascale Ferrand, « Mémoires de Trévoux 1 (1701-1767)», dans Jean Sgard (dir.), Dictionnaire des journaux (1600-1789), Paris, Universitas, 1991, no 889.

${ }^{66}$ Voir « Préface ", Journal britannique, par M. Maty, Docteur en Pbilosopbie \& en Médecine, La Haye, H. Scheurleer Junior, janvier 1750, pp. I-XVI.

${ }^{67}$ Voir «Préface », Bibliothèque impartiale [janvier-février 1750], Leyde, Élie Luzac, 1753 [2 $2^{\text {nde édition] }}, s . p$.

68 Voir Timothée Léchot, " $\mathrm{Si}$ vous m'envoyez de l'etoffe je vous renvoyerai des extraits" : Élie Bertrand et l'art de la transaction journalistique », dans Rossella Baldi (dir.), Élie Bertrand (1713-1797): science, religion et éducation au sein de l'État, Genève, Éditions Slatkine, à paraître.

${ }^{69}$ Voir la lettre de Louis Bertrand à Johann Albrecht Euler, Londres, 8 novembre 1757, University of Tartu, DSpace Repository : http://hdl.handle.net/10062/12612 (31 octobre 2016). L'extrait concerné est publié dans deux livraisons successives de la Nouvelle Bibliothèque germanique : janvier-mars 1758, pp. 132-156; avril-juin 1758, pp. 262-285. Il se présente comme la suite de l'extrait de Louis Bertrand cité supra, note 29.

70 "Préface des éditeurs», Gazette littéraire et universelle de l'Europe, qui contient l'Annonce \& les Extraits des principaux Livres qu'on y met au jour; avec divers morceaux sur l'Agriculture, l'Oeconomie rurale, le Commerce, la Poesie, la Peinture, la Musique \& la Sculpture, \&c. \&c., Lausanne, François Grasset \& Comp., t. 1, 1768, p. V.

71 "Préface », Critique desinteressée des journaux littéraires et des ouvrages des savans, Par une Societé de Gens de Lettres, La Haye, Chrétien Van Lom, janvier-mars 1730, s. p.

${ }^{72}$ Voir « Article I. Journal des Savans, \&c. », Critique desinteressée des journaux littéraires et des ouvrages des savans. Par une Societé de Gens de Lettres, La Haye, Chrétien Van Lom, janvier-mars 1730, pp. 9-17.

73 "Préface", Histoire littéraire de l'Europe : contenant l'Extrait des meilleurs Livres; un Catalogue choisi des Ouvrages nowveaux; les Nouvelles les plus interessantes de la République des Lettres, \& les Pieces fugitives les plus curieuses, La Haye, M. G. de Merville, t. 1, janvier 1726, s. p.

Vol. 8, n 2 | Printemps 2017 
74 «Préface », Nouvelle Bibliothèque on histoire littéraire des principaux Ecrits qui se publient, La Haye, Pierre Paupie, t. 1, octobre 1738, p. 4.

75 «Préface », Bibliothèque germanique ou histoire littéraire de l'Allemagne et des pays du Nord, Amsterdam, Pierre Humbert, t. 1, juillet-septembre 1720, p. VII.

76 [Lettre sans titre], Mémoires secrets de la République des lettres: ou le théatre de la vérité. Par l'Auteur des Lettres Juives, Amsterdam, Jacques Desbordes, 1737, p. 19.

77 Voir «Préface», Histoire des ouvrages des sçavans, Par Monsr. B*** Docteur en Droit, Rotterdam, Reinier Leers, septembre 1687, s. $p$.

${ }^{78}$ Voir « Préface », Journal litéraire, La Haye, T. Johnson, t. 1, mai-juin 1713, pp. VII-XIX.

79 Voir "Avertissement», Bibliothèque angloise, on histoire littéraire de la Grande Bretagne, par M. D. L. R., Amsterdam, Veuve de Paul Marret, t. 1, 1 ère partie, 1717, s. $p$.

80 Voir "Avertissement», Mercure de France, dédié au Roy, Paris, Guillaume Cavelier, Guillaume Cavelier fils, Noel Pissot, janvier 1724, s. p.

81 Voir par exemple les Nouvelles de la République des lettres, éd. cit., p. 3-18.

82 "Idées préliminaires sur la Critique», Journal de littérature, des sciences et des arts; Par M. l'Abbé Grosier (Paris, Bureau du Journal), 1779, p. 14.

83 Alexis Lévrier, «Desfontaines, Marivaux et leurs "petites feuilles" : quelques points de rencontre inattendus », dans Malcolm Cook, Marie-Emmanuelle Plagnol-Diéval (dir.), Critique, critiques au $18^{e}$ siècle, Berne, Peter Lang, 2006, p. 65. Voir aussi du même auteur «Impossible et nécessaire polémique. Les contradictions du journalisme de critique littéraire au XVIII siècle », dans Marie-Hélène Servet, Pierre Servet (dir.), Polémiques en tous genres (XVI ${ }^{e}$-XVIII siècles), Cabiers du GADGES, no 7, 2009, pp. 287-305.

${ }^{84}$ Voir « Article VI. Bibliotheque Italique, ou Histoire Litteraire de l'Italie. Tomes I. II. III. \& IV. A Geneve chez Marc Michel Bousquet \& Compagnie, Libraires \& Imprimeurs, in 8. „, Critique desinteressée des journaux littéraires et des ouvrages des savans, Par une Societé de Gens de Lettres, La Haye, Chrétien Van Lom, janvier-mars 1730, t. 1, janvier-mars 1730, pp. 44-49.

85 «Préface », Bibliothèque italique on histoire littéraire de l'Italie, Genève, Marc-Michel Bousquet \& Compagnie, t. 1, janvier-avril 1728, p. XX.

86 "Préface », Journal britannique, par M. Maty, Docteur en Philosopbie \& en Médecine, La Haye, H. Scheurleer Junior, janvier 1750, p. X.

${ }^{87}$ Denis Diderot, * JOURNALISTE, s. m. (Littérat.), dans Denis Diderot, Jean le Rond D’Alembert (dir.), op. cit., t. 8, Neuchâtel, Samuel Faulche, 1765, p. 898a.

88 "Préface ", Bibliothèque universelle et historique de l'année M. D. C. LXXXVI., Amsterdam, Wolfganc, Waesberge, Boom, van Someren, t. 1, 168[6], s. $p$.

89 "Préface », Essays de littérature pour la connoissance des livres, Paris, Jean Moreau, 15 juillet 1702, s. p. 
90 Le Nouvelliste du Parnasse, on réflexions sur les ouvrages nouveaux, Paris, Chaubert, t. 1, 11e lettre, 1731, p. 255. Voir Alexis Lévrier, «Impossible et nécessaire polémique. Les contradictions du journalisme de critique littéraire au XVIII $I^{e}$ siècle », dans Marie-Hélène Servet, Pierre Servet (dir.), Polémiques en tous genres (XVIT-XVIII siècles), Cabiers du GADGES, no 7, 2009, pp. 289-291.

91 «Préface », Nouvelles de la République des lettres. Seconde Edition revüë \& corrigée par l'Auteur, Amsterdam, Henry Desbordes, mars 1684, s. p.; «Préface ", Histoire des ouvrages des sçavans, Par Monsr. B**** Docteur en Droit, Rotterdam, Reinier Leers, septembre 1687, s. $p$.

92 [Préface sans titre], Journal de politique et de littérature, contenant les principaux Evènemens de toutes les Cours; les Nouvelles de la République des Lettres, \&c., Bruxelles, s. n., t. 1, no 1, 25 octobre 1774, p. 5.

${ }^{93}$ Voir par exemple l'« Avertissement des libraires » de la Bibliothèque raisonnée des ouvrages des savans de l'Europe, Amsterdam, Wetsteins \& Smith, t. 1, juillet-septembre 1728, pp. V-XVII.

94 Voir le sous-chapitre «Les rédacteurs, des guides du public» de Suzanne Dumouchel, Le Journal littéraire en France au dix-buitième siècle. Émergence d'une culture virtuelle, Oxford, The Voltaire Foundation, 2016, pp. 140-146.

${ }_{95}$ «Préface », L'Europe savante, La Haye, A. de Rogissart, t. 1, 1ère partie, janvier 1718, p. XIV.

96 "Préface », L’Europe savante, La Haye, A. de Rogissart, t. 1, 1 ère partie, janvier 1718, p. XVI.

97 «Préface », Histoire littéraire de l'Europe : contenant l'Extrait des meilleurs Livres; un Catalogue choisi des Ouvrages nouveaux; les Nouvelles les plus interessantes de la République des Lettres, \& les Pieces fugitives les plus curieuses, La Haye, M. G. de Merville, t. 1, janvier 1726, s. p.

98 «Préface », Histoire littéraire de l'Europe : contenant l'Extrait des meilleurs Livres; un Catalogue choisi des Ouvrages nouveaux; les Nouvelles les plus interessantes de la République des Lettres, \& les Pieces fugitives les plus curieuses, La Haye, M. G. de Merville, t. 1, janvier 1726, s. p.

99 "Idées préliminaires sur la Critique », Journal de littérature, des sciences et des arts; Par M. l'Abbé Grosier (Paris, Bureau du Journal), 1779, p. 5.

100 «Idées préliminaires sur la Critique», Journal de littérature, des sciences et des arts; Par M. l'Abbé Grosier (Paris, Bureau du Journal), 1779, p. 7.

101 "Idées préliminaires sur la Critique», Journal de littérature, des sciences et des arts; Par M. l'Abbé Grosier (Paris, Bureau du Journal), 1779, p. 17.

102 "Idées préliminaires sur la Critique ", Journal de littérature, des sciences et des arts; Par M. l'Abbé Grosier (Paris, Bureau du Journal), 1779, pp. 24-25.

103 «Avertissement », Le Journal des sçavans, Paris, Jean Cusson, 1702, p. 3.

Vol. 8, n 2 | Printemps 2017

«Le livre et le journal : croisements, prolongements et transformations » 
104 «Préface », Journal litéraire, La Haye, T. Johnson, t. 1, mai-juin 1713, p. IV.

105 «Préface », Journal litéraire, La Haye, T. Johnson, t. 1, mai-juin 1713, p. IV.

106 Ioana Galleron Marasescu, «Van Effen dans ses journaux: le moraliste critique littéraire ", dans Malcolm Cook, Marie-Emmanuelle Plagnol-Diéval (dir.), Critique, critiques au 18 e siècle, Berne, Peter Lang, 2006, p. 43.

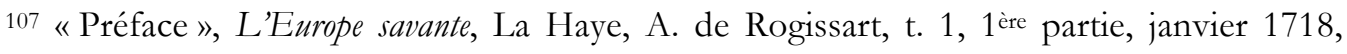
p. XI.

108 «Préface », L'Europe savante, La Haye, A. de Rogissart, t. 1, 1ère partie, janvier 1718, p. XII.

109 «Préface », L’Europe savante, La Haye, A. de Rogissart, t. 1, 1ère partie, janvier 1718, p. XVIII.

110 Voir « Article II. Journal Litteraire; Année 1729. Tome XIII. A la Haye chez P. Gosse \& J. Neaulme", Critique desinteressée des journaux littéraires et des ouvrages des savans, Par une Societé de Gens de Lettres, La Haye, Chrétien Van Lom, janvier-mars 1730, t. 1, janvier-mars 1730, pp. 17-21.

111 Le Nouvelliste du Parnasse, on réflexions sur les ouvrages nouveaux, Paris, Chaubert, t. 1, 11e lettre, 1731, pp. 254-255.

112 «Avertissement des associés a ce journal », Journal encyclopédique, par une societé de gens de lettres, dédié à Son Alt. Ser. \& Emin. Jean-Théodore, Duc de Baviere, Cardinal, Evếque \& Prince de Liege, de Freysing \& Ratisbonne, \&c., Liège, Everard Kints, t. 1, $1^{\text {ère }}$ partie, janvier 1756, p. XI.

113 Suzanne Dumouchel, Le Journal littéraire en France au dix-huitième siècle. Émergence d'une culture virtuelle, Oxford, The Voltaire Foundation, 2016, p. 255.

114 Alexis Lévrier, «Impossible et nécessaire polémique. Les contradictions du journalisme de critique littéraire au XVIII e siècle», dans Marie-Hélène Servet, Pierre Servet (dir.), Polémiques en tous genres (XVI'-XVIII siècles), Cabiers du GADGES, no 7, 2009, pp. 294-295.

115 Alexis Lévrier, «Impossible et nécessaire polémique. Les contradictions du journalisme de critique littéraire au XVIII e siècle», dans Marie-Hélène Servet, Pierre Servet (dir.), Polémiques en tous genres (XVI ${ }^{e}$-XVIII siècles), Cabiers du GADGES, no 7, 2009, p. 294.

116 «Préface », Journal litéraire, La Haye, T. Johnson, t. 1, mai-juin 1713, p. XI.

117 Voir «Préface", Histoire littéraire de l'Europe : contenant l'Extrait des meilleurs Livres; un Catalogue choisi des Ouvrages nowveaux; les Nouvelles les plus interessantes de la République des Lettres, \& les Pieces fugitives les plus curieuses, La Haye, M. G. de Merville, t. 1, janvier 1726, s. p.

118 Partagée par les journalistes, l'impression d'une croissance exponentielle des nouveaux périodiques savants ou littéraires correspond à une réalité mesurable jusque dans les années 1750 environ. Voir la postface de Jean Sgard (dir.), Dictionnaire des joumaux (16001789), Oxford, The Voltaire Foundation, 1999, pp. 1131-1140.

Vol. 8, nº 2 | Printemps 2017

«Le livre et le journal : croisements, prolongements et transformations » 
119 [Préface sans titre], La Feuille nécessaire, contenant divers détails sur les sciences, les lettres et les arts, Paris, Michel Lambert, 1759, p. 3.

120 "Discours préliminaire », Journal des journaux ou précis des principaux Ouvrages Périodiques de l'Europe, par une Société de gens de Lettres, dédié à son Altesse sérénissime électorale palatine, Mannheim, Imprimerie électorale, t. 1, 1760, p. XIV.

121 Voir "Avertissement», Essai d'un nouveau journal intitulé, Le Littérateur impartial, ou précis des ouvrages périodiques, La Haye, s. n., 1760, pp. V-X.

122 Voir Eugène Hatin, Histoire politique et littéraire de la presse en France. Avec une introduction historique sur les origines du journal et de la bibliographie générale des journaux depuis leur origine, Paris, De Broise, Poulet-Malassis, 1858-1861, t. 3.

123 Jean Rousset de Missy à Prosper Marchand, s. l., 21 août [1752], dans Christiane Berkens-Stevelinck, Jeroom Vercruysse (éd.), Le métier de journaliste au dix-buitième siècle. Correspondance entre Prosper Marchand, Jean Rousset de Missy et Lambert Ignace Douxfils, Oxford, The Voltaire Foundation, 1993, p. 152. Dans le quartier parisien de Montfaucon se trouvait depuis le Moyen Âge un célèbre gibet; on y rencontrait aussi des boyaudiers dont une partie du travail consistait à dégraisser les viscères des animaux pour en faire des cordes.

124 «Préface », Bibliothèque impartiale, [janvier-février 1750], Leyde, Élie Luzac, 1753 [2nde édition], s. $p$.

125 Voir la synthèse et la bibliographie proposées par Anne-Marie Chouillet, "Denis Diderot (1713-1784)», dans Jean Sgard (dir.), Dictionnaire des journalistes (1600-1789), Oxford, The Voltaire Foundation, 1999, no 240.

126 Voir la lettre de Denis Diderot à Louise Henriette Volland, dite Sophie Volland, Paris, 26 mai 1759, dans Denis Diderot, Lettres à Sophie Volland 1759-1774, Marc Buffat, Odile Richard-Pauchet (éd.), Paris, Non Lieu, 2010, p. 31.

127 Sur l'activité critique et intellectuelle de Chaillet, voir notamment la monographie de Charly Guyot, Henri-David de Chaillet critique littéraire 1751-1823, Neuchâtel, Imprimerie Paul Attinger, 1946.

128 Voir les travaux d'Alexis Lévrier sur les différents «spectateurs» et notamment "D'Addison à Marivaux : le modèle du Spectator à l'épreuve des contraintes françaises", Études Épistémè, no 26, 2014 : episteme.revues.org/306 (31 octobre 2016).

129 Sur l'impact de Fréron et de ses journaux, voir notamment Jean Balcou, "L'Année littéraire (1754-1776) ", Jean Sgard (dir.), Dictionnaire des journaux (1600-1789), Oxford, The Voltaire Foundation, 1999, no 118; et les études rassemblées dans Jean Balcou (dir.), Élie Fréron. Polémiste et critique d'art, Rennes, Presses universitaires de Rennes, 2001.

${ }^{130}$ Le Nouvelliste du Parnasse, on réflexions sur les ouvrages nouveaux, Paris, Chaubert, t. 1, $12^{\mathrm{e}}$ lettre, 1731, p. 278. Voir aussi Suzanne Dumouchel, Le Journal littéraire en France au dix-buitième siècle. Émergence d'une culture virtuelle, Oxford, The Voltaire Foundation, 2016, pp. 256-257; Alexis Lévrier, Les journaux de Marivaux et le monde des «spectateurs », Paris, Presses 
de l'Université Paris-Sorbonne, 2007, pp. 140-141; et Alexis Lévrier, «Desfontaines, Marivaux et leurs "petites feuilles": quelques points de rencontre inattendus ", dans Malcolm Cook, Marie-Emmanuelle Plagnol-Diéval (dir.), Critique, critiques an $18^{e}$ siècle, Berne, Peter Lang, 2006, pp. 75-77.

131 «Avertissement au sujet du nouvel Ouvrage Périodique, intitulé, l'Année Littéraire, par M. Fréron, des Académies d'Angers, de Montauban \& de Nancy ", L'Année littéraire ou suite des Lettres sur quelques Ecrits de ce Temps, par M. Fréron des Académies d'Angers, de Montauban \& de Nancy, Amsterdam, Michel Lambert, t. 1, 1754, pp. 5-6.

132 Sur le devoir de neutralité dans Le Nouvelliste du Parnasse et dans l'Année littéraire, et sur la manière dont leurs journalistes respectifs l'interprètent, voir Suzanne Dumouchel, Le Journal littéraire en France au dix-buitième siècle. Émergence d'une culture virtuelle, Oxford, The Voltaire Foundation, 2016, pp. 83-86.

\section{Bibliographie}

Christiane Berkens-Stevelinck, Jeroom Vercruysse (éd.), Le métier de journaliste au dix-huitième siècle. Correspondance entre Prosper Marchand, Jean Rousset de Missy et Lambert Ignace Douxfils, Oxford, The Voltaire Foundation, 1993.

Hans Bots, Jan de Vet (éd.), Stratégies journalistiques de l'Ancien Régime. Les préfaces des "Journaux de Hollande », 1684-1764. Introduction et édition annotée de trente-buit préfaces de journaux savants, avec un index des noms, Amsterdam, Utrecht, APA-Holland University Press, 2002.

Malcolm Cook, Marie-Emmanuelle Plagnol-Diéval (dir.), Critique, critiques au $18^{e}$ siècle, Berne, Peter Lang, 2006.

Élisabeth Décultot (dir.), Lire, copier, écrire. Les bibliothèques manuscrites et leurs usages au XVIII siècle, Paris, CNRS Éditions, 2003.

Suzanne Dumouchel, Le Journal littéraire en France au dix-buitième siècle. Émergence d'une culture virtuelle, Oxford, The Voltaire Foundation, 2016.

Jens Häseler, "Extraits - abrégés - encyclopédies », dans Jean-Daniel Candaux, Alain Cernuschi, Clorinda Donato, Jens Häseler (dir.), L'Encyclopédie d'Yverdon et sa résonance européenne. Contextes - contenus - continuités, Genève, Éditions Slatkine, 2005, pp. 277-288.

Claude Labrosse, Pierre Rétat, L’instrument périodique. La fonction de la presse au XVIII siècle, Lyon, Presses universitaires de Lyon, 1985.

Steve Larkin (éd.), Correspondance entre Prosper Marchand et le marquis d'Argens, Oxford, The Voltaire Foundation, 1984. 
Alexis Lévrier, Les journaux de Marivaux et le monde des «spectateurs », Paris, Presses de l’Université Paris-Sorbonne, 2007.

Alexis Lévrier, «Impossible et nécessaire polémique. Les contradictions du journalisme de critique littéraire au XVIII ${ }^{\mathrm{e}}$ siècle », dans Marie-Hélène Servet, Pierre Servet (dir.), Polémiques en tous genres (XVI'-XVIII siècles), Cabiers du GADGES, $\mathrm{n}^{\circ}$ 7, 2009, pp. 287-305.

Jeanne Peiffer, Jean-Pierre Vittu, «Les journaux savants, formes de la communication et agents de la construction des savoirs $\left(17^{\mathrm{e}}-18^{\mathrm{e}}\right.$ siècles) $)$, Dix-buitième siècle, $\mathrm{n}^{\circ}$ 40, 2008, pp. 281-300.

Jean Sgard (dir.), Dictionnaire des journalistes (1600-1789), Oxford, The Voltaire Foundation, 1999.

Jean Sgard (dir.), Dictionnaire des journaux (1600-1789), Paris, Universitas, 1991. 